\title{
Macrophage-derived cholesterol contributes to therapeutic resistance in prostate cancer
}

Asmaa El-Kenawi ${ }^{*}$, William Dominguez-Viqueira², Min Liư ${ }^{3}$, Shivanshu Awasthi ${ }^{4}$, Aysenur Keske ${ }^{1}$, KayLee K. Steiner ${ }^{1}$, Leenil Noel ${ }^{1}$, Jasreman Dhillon ${ }^{5}$, Robert J. Gillies ${ }^{6}$, Kosj Yamoah ${ }^{4,7}$, Xiaoqing $\mathrm{Yu}^{8}$, John Koomen ${ }^{3,9}$, Robert A. Gatenby ${ }^{10}$ and Brian Ruffell ${ }^{1,11^{*}}$

${ }^{1}$ Department of Immunology

2 Small Animal Imaging Lab

${ }^{3}$ Proteomics and Metabolomics Core

${ }^{4}$ Department of Cancer Epidemiology

${ }^{5}$ Department of Pathology

${ }^{6}$ Department of Cancer Physiology

${ }^{7}$ Department of Radiation Oncology

${ }^{8}$ Department of Biostatistics and Bioinformatics

9 Department of Molecular Oncology

10 Department of Radiology

${ }^{11}$ Department of Breast Oncology, H. Lee Moffitt Cancer Center and Research Institute, Tampa, Florida 33612, USA.

*Correspondence: AsmaaKenawy@mans.edu.eg, (A.E.), Brian.Ruffell@moffitt.org (B.R.)

Running Title: Macrophages regulate androgen receptor activation in prostate cancer

Keywords: Prostate cancer, macrophages, androgens, androgen receptor, biosynthesis, metabolism

Contact: Brian Ruffell, Ph.D., H. Lee Moffitt Cancer Center, 12902 Magnolia Drive SRB-2, Tampa, FL, 33612, Voice: 813-745-6305, Email: Brian.Ruffell@moffitt.org 


\section{Abstract}

Tumor-associated macrophages are key immune cells associated with cancer progression. Here we sought to determine the role of macrophages in castration-resistant prostate cancer (CRPC) using a syngeneic model that reflected the mutational landscape of the disease. A transcriptomic analysis of CRPC tumors following macrophage depletion revealed lower molecular signatures for steroid and bile acid synthesis, indicating potential perturbation of cholesterol metabolism. Since cholesterol is the precursor of the five major classes of steroid hormones, we reasoned that macrophages were regulating androgen biosynthesis within the prostate tumor microenvironment. Indeed, macrophage depletion reduced the levels of androgens within prostate tumors and restricted androgen receptor (AR) nuclear localization in vitro and in vivo. Macrophages were cholesterol rich and had the ability to transfer cholesterol to tumor cells in vitro, and AR nuclear translocation was inhibited by activation of Liver $X$ Receptor (LXR)- $\beta$, the master regulator of cholesterol homeostasis. Finally, combining macrophage depletion with androgen deprivation therapy increased survival, supporting the therapeutic potential of targeting macrophages in CRPC. 


\section{Introduction}

Prostate cancer is the second leading of cancer death in men in the United States. Androgen hormones drive prostate cancer cell proliferation and progression by activating the androgen receptor (AR). Given their tumor-promoting role, reducing androgen levels or signaling by using Androgen Deprivation Therapy (ADT) is the standard approach to treat advanced prostate cancer $(1,2)$. In most cases ADT induces tumor regression; however, resistance eventually develops and the disease relapses as Castration-Resistant Prostate Cancer (CRPC) $(3,4)$. Androgen-AR signaling persists in many cases of CRPC via alterations in the AR gene, deregulation of AR binding-proteins (2) and/or intratumoral synthesis of androgens (5); thus, both androgen synthesis inhibitors and AR inhibitors can extend survival (6). However, while much is known regarding the intrinsic mechanisms of AR reactivation within tumor cells, little is understood regarding the contributions of the tumor microenvironment in therapeutic resistance in CRPC.

Macrophages are the most abundant immune subpopulation in many solid tumors, wherein they adopt a maladaptive phenotype and are commonly known as tumor-associated macrophages (TAMs) (7). In addition to their role in creating the immunosuppressive microenvironment in tumors (8), TAMs can promote tumor growth and resistance to therapy through the release of cytokines and angiogenic mediators $(9,10)$. More recently, TAM-secreted metabolites have emerged as a driver of drug resistance. TAM-derived pyrimidines diminished the efficacy of gemcitabine in pancreatic cancer through molecular competition during drug uptake, while in a model of colon cancer, TAMs were involved in the production of glucocorticoids and impaired the efficacy of immune checkpoint blockade (11). Whether macrophage-tumor cell metabolic interactions dictate therapeutic responses in prostate cancer is unknown.

Here we sought to determine the role of macrophages in CRPC using a murine model that recapitulated the high AR signaling and mutational landscape of prostate cancer. We developed orthotopic systems derived from an inducible, autochthonous model of Trp53/Pten-deficiency with or without the most common ETS rearrangement, TMPRSS2/ERG fusion. We then performed transcriptomic and metabolomic characterization of prostate tumors following macrophage depletion. Combined with efficacy studies and in vitro experiments, our data demonstrate that macrophages promote AR signaling and castration resistance via enhanced tumor cell cholesterol influx and androgen biosynthesis. 


\section{Materials and Methods}

Animal models: All mice were maintained in accordance with Institutional Animal Care and Use Committee (IACUC) standards followed by the Moffitt Cancer Research Center (Tampa, Florida). The conditional model of prostate cancer was established by crossing Trp53 ${ }^{\operatorname{loxP}}$ (Trp53 ${ }^{\mathrm{tm} 1 \mathrm{Brn}} / \mathrm{J} ; \mathrm{JAX}$

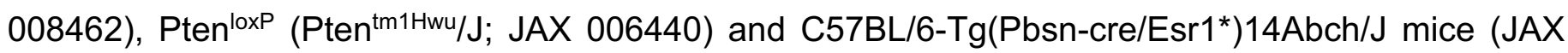
020287), with or without expression of the TMPRSS2/ERG fusion gene under control of the Rosa26

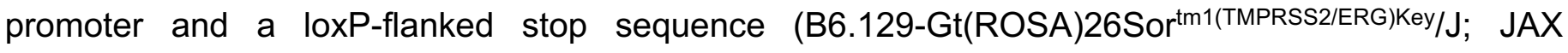
024512). The Pbsn-cre/Esr1* transgene allows inducible Cre-mediated recombination in the mouse prostate following ad libitum dosing of $500 \mathrm{mg} / \mathrm{kg}$ tamoxifen-containing chow for 4 weeks(12). Dual Abca1/Abcg1-floxed mice (B6.Cg-Abca1tm1Jp Abcg1tm1Tall/J; JAX 021067) were crossed with

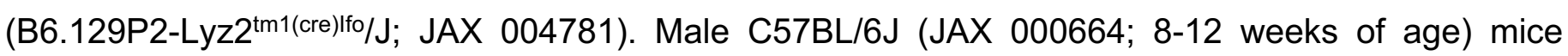
purchased from The Jackson Laboratory were utilized for orthotopic prostate cancer models and bone marrow isolation. Mice were treated with $0.6 \mathrm{mg}$ Lupron Depot (AbbVie Inc. North Chicago) by subcutaneous injection. aCSF1 (clone 5A1, BioXCell) was administrated intraperitoneally at 1 $\mathrm{mg} / \mathrm{mouse}$, followed by $0.5 \mathrm{mg} / \mathrm{mouse}$ every 5 days (13).

Magnetic resonance Imaging (MRI): All MRI experiments were done in a 7T horizontal magnet (Agilent-Technologies) and Bruker electronics (BioSpec AV3HD), using a $35 \mathrm{~mm}$ birdcage coil (Doty Scientific). Mice were anesthetized with $2 \%$ isoflurane in $\mathrm{O}_{2}$ during data acquisition. T2-weighted axial images were acquired with a TurboRARE-sequence (TR/TE= 2446/42 ms, FOV $=35 \times 35 \mathrm{~mm}^{2}$, image size $256 \times 256$ and 21 slices of $1.3 \mathrm{~mm}$ thickness and non-fat-suppression). Tumor volume analyses were performed by manual segmentation using in-house written scripts in Matlab (Mathworks Inc.).

Cell lines: Male-derived murine RAW 264.7 macrophages, TRAMP-C2 and TRAMP-C3 prostate cancer cell lines were purchased from ATCC, maintained and cultured according to their suggested protocols. ERG- (PT-83, PT-25, PT-09) and ERG ${ }^{+}$(PTE-24, PTE-82) syngeneic cell lines (all Ptenand Trp53-deficient) were generated from tamoxifen treated male mice bearing end-stage prostate tumors as described by others (14). All cell lines lacked expression of CD45 or smooth muscle actin ( $\alpha-S M A)$, expressed variable levels of androgen receptor, and expressed detectable keratin 14 and 18. Cells were maintained in Dulbecco modified Eagle's minimal essential medium (DMEM), $25.0 \mathrm{mM}$ glucose, $25.0 \mathrm{mM}$ HEPES supplemented with 10\% fetal calf serum (FCS), $2 \%$ penicillin/streptomycin, $0.005 \mathrm{mg} / \mathrm{ml}$ bovine insulin and $10 \mathrm{nM}$ dehydroisoandrosterone (DHEA). Subculturing was performed when cells reached $90 \%$ confluency and only passage numbers below 10 were used for in vivo experiments. 
Primary cells: Bone marrow-derived macrophages (BMDMs) were generated as described previously $(15,16)$. In brief, bone marrow was flushed from femurs and tibias of male C57BL/6J mice and cultured at $37^{\circ} \mathrm{C}$ for 6-7 days in complete macrophage medium (Dulbecco modified Eagle's minimal essential medium (DMEM), $25.0 \mathrm{mM}$ glucose, $25.0 \mathrm{mM}$ HEPES, supplemented with 10\% fetal calf serum (FCS), $2 \%$ penicillin/streptomycin, and $20 \mathrm{ng} / \mathrm{ml} \mathrm{M-CSF} \mathrm{(R \& D} \mathrm{Systems).}$

Flow cytometry: Mice were cardiac perfused with PBS containing $10 \mathrm{U} / \mathrm{ml}$ heparin to clear peripheral blood followed by surgical excision of prostate tissue and/or tumors. Single cell suspensions were then prepared by incubating minced tissue in $1 \mathrm{mg} / \mathrm{ml}$ collagenase (Roche) and $50 \mathrm{U} / \mathrm{ml}$ DNase I (Roche) at $32^{\circ} \mathrm{C}$ with agitation for $30 \mathrm{~min}$. The resulting cell suspensions was passed through a 70 $\mu \mathrm{m}$ cell strainer and washed once with FBS-containing DMEM followed by once with PBS. Single cell suspensions were then incubated in ACK Lysing Buffer at RT for 3-5 min. Single cell suspensions were then stained immediately or stored in freezing medium (5\% charcoal-stripped serum [CSS], 10\% DMSO and $85 \% \mathrm{DMEM}$ ) at $-80^{\circ} \mathrm{C}$ for later analysis. Staining protocol involved incubation with live/dead (L/D) fixable fluorescent reactive dyes (Molecular Probes) in PBS for 10 min. After washing, cells were incubated for $30 \mathrm{~min}$ at $4^{\circ} \mathrm{C}$ in $100 \mu$ staining buffer (PBS, 2\% BSA) containing antibodies. For intracellular lipid staining, single cell suspensions were incubated with $0.25 \mu \mathrm{M}$ Bodipy $^{\mathrm{TM}} 493 / 503$ in PBS for 15 min at RT. Paraformaldehyde Solution, 4\% in PBS (Affymetrix) was used to fix cells. Data was recorded on a LSR II Flow Cytometer (BD Biosciences) and analysis completed using FlowJo software.

LDL uptake: Tumor cells were seeded in culture plates and starved for $48 \mathrm{~h}$ in $5 \%$ CSS DMEM.

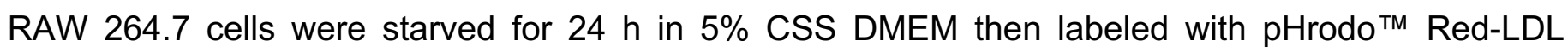
(L34356) 1:2000 for the last $3 \mathrm{~h}$. Tumor cells were then co-cultured with labeled RAW 264.7 cells for another $24 \mathrm{~h}$ in serum-free medium. Samples were then processed for flow cytometry by staining with CD45-APC and DAPI. Cells were mixed just prior to acquisition on the LSRII for the 0 min incubation.

Co-culture: 3000 tumor cells were seeded in each well of 96 well plate, then 1000 macrophages were added on the following day in DMEM (phenol red free, contains $25 \mathrm{mM}$ glucose and $25 \mathrm{mM}$ HEPES) supplemented with charcoal stripped serum (CSS) with or without serial dilutions of enzalutamide $(10,20,40,80 \mu \mathrm{M})$. Time lapse microscopy measurements were performed every 6-8 $\mathrm{h}$ in phase-contrast white light using Incucyte Zoom system (Essen Bioscience). Percent confluency was calculated for each time point using a tailored algorithm for each cell line, and data were normalized to initial time point of each well. 
Confocal immunofluorescence: Cells on chamber slides were washed twice with PBS, fixed with Paraformaldehyde Solution, 4\% in PBS for $20 \mathrm{~min}$ and permeabilized with $0.1 \%$ Triton X-100 for 5 min. Cells were washed twice with PBS, blocked with $2 \%$ BSA in PBS for $1 \mathrm{hr}$ and subsequently incubated with anti-androgen receptor antibody $(1: 400, \mathrm{Abcam})$ at $4^{\circ} \mathrm{C}$ overnight. Cells were washed 3 times with PBS and incubated with appropriate fluorescent-labeled secondary antibodies at RT for 1 hr, followed by incubating with F4/80-APC (1:400) for $1 \mathrm{~h}$ RT in the case of macrophage co-culture experiments. VECTASHIELD® Antifade containing DAPI was used as mounting medium. Vybrant ${ }^{\mathrm{TM}}$ Alexa Fluor ${ }^{\text {TM }} 555$ Lipid Raft Labeling Kit was utilized according to the manufacturer's instructions. Images were visualized using Leica TCS SP8 laser scanning microscope (Leica Microsystems). The nucleus to cytoplasm AR intensity ratio was plotted using the basic (generic) $R$ command hist using $R$ studio version 4.0.3. A smooth curve was fitted to the histogram using kernel density estimation computed by the density function.

Histology and immunohistochemistry (IHC): Histological specimens were embedded in paraffin, sectioned (4 $\mu \mathrm{m}$ slices) and left unstained or stained with haematoxylin \& eosin (H\&E). For immunohistochemistry, slides were stained using a Ventana Discovery XT automated system (Ventana Medical Systems). Briefly, slides were deparaffinized on the automated system with EZ Prep solution (Ventana). Enzymatic retrieval method was used in Protease 1 (Ventana). The rabbit primary antibodies that react to AR (1:200, Abcam), F4/80 (1:100, Abcam), $\alpha-S M A$ (1:250, Abcam), Ly6G (1:100, Biolegend), CD3 (1:200, Abcam), Ki67 (1:300, Abcam), in Dako antibody diluent (Agilent) and incubated for $60 \mathrm{~min}$. The Ventana OmniMap Anti-Rabbit Secondary Antibody was used for $8 \mathrm{~min}$. The detection system used was the Ventana ChromoMap kit, and slides were then counterstained with hematoxylin, followed by dehydration and cover-slipping. Histology slides were scanned using the Aperio ${ }^{\mathrm{TM}}$ ScanScope XT with a 200X-(0.8NA) objective lens at a rate of 5 minutes per slide via Basler tri-linear-array. Images were analyzed with Aperio eSlide Manager software using nuclear (AR), membrane (F4/80), or pixel (SMA, Ly6G, Ki67) detection algorithms. Intensity thresholds in the default algorithms for hematoxylin recognition were adjusted to identify faintly stained nuclei. In addition, the positive staining categories (weak, moderate, strong) on the default nuclear detection algorithm were adjusted to account for weaker staining. All Intensity thresholds remained consistent for each image within the respective biomarker group.

LC-MS/MS Sample Preparation: Stock mixtures of stable isotope-labeled standards were prepared in either methanol or acetonitrile at a concentration of $10 \mathrm{mg} / \mathrm{mL}$ and then diluted to lower concentrations. Tumor tissues were collected and stored at $-80^{\circ} \mathrm{C}$ until use. Aliquots of tumor tissue ( 50-250 mg) were weighed and placed in Eppendorf microcentrifuge tubes. Samples were kept on ice throughout the processing steps. Each sample was spiked with $10 \mu \mathrm{L}$ of 9 androgen internal 
standard mixtures at a concentration of $5 \mathrm{ng} / \mathrm{mL}$ for $\mathrm{T}\left(2,3,4-{ }^{13} \mathrm{C}_{3}\right)$, Prog $\left(2,3,4-{ }^{13} \mathrm{C}_{3}\right), \operatorname{AED}\left(2,3,4-{ }^{13} \mathrm{C}_{3}\right)$ and DOC (2,2,4,6,6,17a,21,21-D $), 50 \mathrm{ng} / \mathrm{mL}$ (DHT $\left(2,3,4-{ }^{13} \mathrm{C}_{3}\right), F\left(9,11,12,12-\mathrm{D}_{4}\right)$ and A5-diol $\left(16,16,17-D_{3}\right)$, and $500 \mathrm{ng} / \mathrm{ml}$ for $\operatorname{DHEA}\left(2,2,3,4,4,6-\mathrm{D}_{6}\right)$ and Preg $\left(20,21-{ }^{13} \mathrm{C}_{2} ; 16,16-\mathrm{D}_{2}\right)$. After an hour, each sample was re-suspended in PBS, then 0.9-2.0 mm blend stainless steel beads (Next Advance, Troy, NY) were added. The volume ratio of tissue, PBS, and beads is 1:2:1. Samples were homogenized at speed 8 for 5 minutes at $4^{\circ} \mathrm{C}$ (Bullet Blender, Next Advance, Troy, NY) and transferred to a $5 \mathrm{~mL}$ glass centrifuge tube for Liquid-Liquid Extraction (LLE)(17) followed by Solid Phase Extraction (SPE) ${ }^{(18)}$. Briefly, $2 \mathrm{~mL}$ of MTBE was added to the glass centrifuge tube with sample homogenate in it and then vortexed for 30 seconds. All samples were centrifuged at 2,000 $\mathrm{g}$ for 10 minutes at $5^{\circ} \mathrm{C}$ to separate the organic and aqueous layers. The top organic layer was decanted, and the remaining androgens from the aqueous phase were extracted two more times. The pooled MTBE androgen extract was dried under nitrogen (5 psi) using Reacti-Vap ${ }^{\text {TM }}$ Evaporators (TS 18826, Thermo Fisher Scientific, Waltham, MA) in a fume hood at room temperature. The androgen pellet was re-dissolved in $2 \mathrm{~mL}$ of aqueous 20\% methanol and loaded into Isolute C18 cartridges (200 mg/3 $\mathrm{mL}$ ), which were conditioned with $5 \mathrm{~mL}$ methanol and followed by $3 \mathrm{~mL}$ water. After loading the sample and washing the cartridges with $2 \mathrm{~mL}$ of aqueous $10 \%$ methanol, the androgens were eluted with $2 \mathrm{~mL} 100 \%$ methanol. This eluate was dried and reconstituted with $50 \mu \mathrm{L}$ or $100 \mu \mathrm{L}$ of aqueous $50 \%$ methanol depending on the amount of tissue for each sample. The concentrated extract was transferred to an LC-MS autosampler vial (Fisher Scientific, Hampton, NH) and injected right away.

LC-MS/MS: Liquid Chromatography-Mass Spectrometry (LC-MS) was performed using a UHPLC (Vanquish, Thermo Scientific) interfaced with a $Q$ Exactive HF hybrid quadrupole-Orbitrap mass spectrometer (Thermo Scientific, San Jose, CA). Chromatographic separation was conducted on an Accucore C18 column (2.1 mm ID x $100 \mathrm{~mm}$ in length with $2.6 \mu \mathrm{m}$ particle size) maintained at $40^{\circ} \mathrm{C}$. Separation was achieved using mobile phases $A\left(100 \% \mathrm{H}_{2} \mathrm{O}\right.$ with $0.1 \%$ formic acid) and $\mathrm{B}(100 \%$ $\mathrm{MeOH}$ with $0.1 \%$ formic acid). The gradient was programmed as follows: 2 minutes at $30 \% \mathrm{~B}$, then ramping from $30 \%$ B to $90 \%$ B over 12 minutes, washing with $90 \%$ B for 2 minutes, returning to $30 \%$ $B$ over 0.1 minutes and re-equilbrating for 3.9 minutes for a total run time of 20 minutes. The flow rate was set to $0.250 \mathrm{~mL} / \mathrm{min}$ except $16.1-19 \mathrm{~min}$, where the flow rate was increased to $0.450 \mathrm{~mL} / \mathrm{min}$ for re-equilibration. For MS, parallel reaction monitoring (PRM) was used for the detection and quantification. The instrument settings were as follows: sheath gas 50, auxiliary gas 10, sweep gas 1 , spray voltage $3.5 \mathrm{kV}$, capillary temperature $325^{\circ} \mathrm{C}$, S-lens RF level 50 , auxiliary gas temperature $350^{\circ} \mathrm{C}$, resolution $30,000, \mathrm{AGC}$ target $2 \mathrm{E} 5$, and isolation window width $1.5 \mathrm{~m} / \mathrm{z}$ with an offset of 0.4 $\mathrm{m} / \mathrm{z}$. The data analysis is performed using XCalibur. A processing method was created based on the precursors and fragments listed in Fig. S2. The $\mathrm{m} / \mathrm{z}$ and RT tolerance values were 5 ppm and 0.05 min. The sum of the peak heights of all the fragments for each analyte is used for quantification. To 
calculate the amounts of androgen in the sample, the ion signal for the endogenous androgen was divided by the ion signal for the corresponding SIS and multiplied by the amount of SIS (in pg) spiked into each sample that was injected on column. To enable more effective comparison between samples, final results are normalized by protein amount and expressed as pg androgen/mg protein.

RNA sequencing: Mice bearing orthotopic prostate tumors were cardiac perfused with PBS (without heparin), following by resection of tumors and immediate freezing in liquid nitrogen. RNA from prostate tumor tissue was extracted using RNeasy® Plus Mini kit (Qiagen) according to manufacturer's protocol with on-column DNase digestion and screened for quality on an Agilent Tape-Station (Agilent Technologies, Inc., Wilmington DE). RNA-sequencing libraries were prepared using the NuGen Ovation Mouse RNA-Seq Kit System (Tecan US, Inc., Morrisville, NC). Briefly, 100 ng of DNase treated RNA was used to generate cDNA and a strand-specific library following the manufacturer's protocol. Library molecules containing ribosomal RNA sequences were depleted using the NuGen Mouse AnyDeplete probe-based enzymatic process. The final libraries were assessed for quality on the Agilent TapeStation (Agilent Technologies, Inc., Wilmington DE), and quantitative RT-PCR for library quantification was performed using the Kapa Library Quantification Kit (Roche Sequencing, Pleasanton, CA). The libraries were sequenced on the Illumina NextSeq 500 v2 sequencer with a 75-base paired-end run in order to generate about 40 million read pairs per sample.

RNA-seq analysis: The raw reads were first assessed for quality using FastQC (http://www.bioinformatics.babraham.ac.uk/projects/fastqc/) and then trimmed by cutadapt(19) (version 1.8.1) to remove reads with adaptor contaminants and low-quality bases. Read pairs with either end too short (<25bps) were discarded from further analysis. Trimmed and filtered reads were aligned to the GRCm38 mouse reference genome using STAR(20) (version 2.5.3a). Unmapped reads were further aligned to GRCh37 human reference genome. Uniquely mapped reads were counted by htseq-count(21) (version 0.6.1) using Gencode m21 annotation for mouse genome and Gencode v30 annotation for human gene ERG. Differential expression analysis was performed using DESeq2 taking into account of RNA composition bias(22). Genes with fold-change $>2$ and false discovery rate (FDR) q-values< 0.05 were considered differentially expressed. For each differential expression analysis comparing treated groups vs. IgG group, mouse genes were converted to their human homologs and ranked based on -log10(p-value)*(sign of log2(fold-change)). The ranked gene list used to perform pre-ranked gene set enrichment analysis (GSEA(23) version 4.0.2) to assess enrichment of hallmarks, curated gene sets, and gene ontology(24) terms in MSigDB(23). The resulting normalized enrichment score (NES) and FDR controlled p-values were used to assess the transcriptome changes induced by Lupron and $\alpha-C S F 1$. 
Real-time quantitative PCR: RNA was extracted using RNeasy isolation kit (Qiagen). Real-time quantitative PCR (RT-qPCR) was then carried out using TaqMan ${ }^{\text {TM }}$ RNA-to-CT ${ }^{\text {TM }}$ 1-Step Kit (Thermo Fisher Scientific). PCR was performed using individual TaqMan Assays with catalogue numbers provided in supplementary table 1 with searchable Research Resource Identifier (RRID). Relative Expression/Fold Change was calculated by using $2^{\Delta \mathrm{Ct}}$ method where $\Delta \mathrm{Ct}=$ Average Ct Gene of Interest - Average Ct of Tbp as Endogenous Control.

TCGA PRAD analysis: The correlation of macrophage-related genes and de novo cholesterol biosynthesis in a prostate cancer cohorts was computed using level 3 gene expression estimates from the RNA-Sequencing in the TCGA PRAD (Prostate Adenocarcinoma Data Set) database, extracted, and hosted by cBioPortal for Cancer Genomics (www.cbioportal.org).

GRID registry: To evaluate the clinical relevance of macrophages in therapeutic resistance to ADT we utilized 635 prostatectomy tumor samples with whole transcriptome data from the Decipher GRID $^{\text {TM }}$ registry data (NCT02609269, institutional review board approved)(25). Gene expression markers of tumor macrophages were used to evaluate their correlation with the ADT response score, a validated gene expression signature used in the prediction of postoperative ADT response, as has been described(26).

Statistical Analysis: Unless otherwise indicated, unpaired t-test was used to facilitate comparisons between two group means. For multiple group comparisons two-way ANOVA was used. Data points represent biological or technical replicates and are shown as the mean \pm SEM or SD as indicated in the figure legends. Intergene correlation matrix with hierarchical clustering, based on spearman correlation coefficient, was used determine correlation clusters. All statistical tests were two sided and are shown as ${ }^{*} p<0.05,{ }^{* *} p<0.01,{ }^{* * *} p<0.001$ (described in each figure legend). Statistical analysis was carried out using R studio v3.5.0 and GraphPad PRISM 7 software. 


\section{Results}

\section{Development of syngeneic mouse models of prostate cancer}

Prostate cancers often harbor recurrent rearrangements of the E26 Transformation-Specific (ETS) family of transcription factors. Among these, fusion of ETS-related gene (ERG) to the membrane protease TMPRSS2 (TMPRSS2-ERG) represents the most common structural rearrangement in

prostate cancer (27). We established a mouse model that reflects the mutational landscape of aggressive prostate cancer by crossing Trp53 ${ }^{\text {lox }}, P t e n^{\text {loxP }}$ and Pbsn-cre/Esr1* mice, with or without the TMPRSS2/ERG fusion gene (Fig. 1A). Cre-mediated recombination was confirmed in the mouse prostate 2 months following the initiation of tamoxifen, as shown by immunohistochemistry staining of

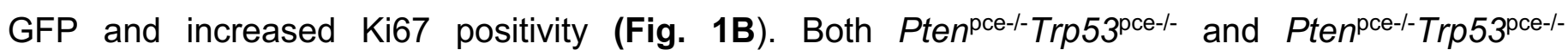
TMPRSS2-ERG ${ }^{\text {pce+ }}$ mice began to develop tumors after 6 months, as detected by MRI, with all mice developing tumors within 12 months (Fig. 1C). In line with the putative oncogenic role of ETS fusions in prostate cancer(28), there was an increase in tumor incidence and progression, and reduced survival in TMPRSS2-ERG ${ }^{\text {pce }}{ }^{+}$mice (Fig. 1C). Neoplastic progression at 6 and 12 months (Fig. 1D) was marked by higher Ki67 positivity and reduced presence of SMA ${ }^{+}$fibromuscular stroma in both genotypes (Fig. S1A-B). Early tumor lesions were androgen driven as mice (4-month post tamoxifen) treated with Lupron, a gonadotropin-releasing hormone analogue that reduces systemic testosterone levels, displayed decreased tumor lesion size in both genotypes, as shown by representative MRI T2weighted images (Fig. 1E, S1C-D). From end stage mice we generated a series of syngeneic TMPRSS2-ERG' (denoted as PTE) and TMPRSS2-ERG- (denoted as PT) prostate cancer cell lines that expressed varying levels of cytokeratin 8, 14, and 18 (Fig. 1F) as well as AR (Fig. 1G). As expected, Pten-deficiency resulted in sensitivity to the PI3K inhibitor GDC-941 (Fig. S1E). AR nuclear translocation was observed following the addition of dihydrotestosterone (DHT) in all cell lines tested (Fig. 1H). However, cell lines displayed differential sensitivity to enzalutamide, including 3 lines (PTE82, PT-09, PT-25) that survived even in the presence of $80 \mu \mathrm{M}$ enzalutamide, thereby representing models of CRPC (Fig. 1I).

\section{Macrophages are associated with cholesterol transport and androgen synthesis}

Both the PT-09 and PTE-82 CRPC cell lines established orthotopic tumors following injection into the prostate glands of C57BL6/J mice. Critically, $\mathrm{F} 4 / 80^{+}$macrophages formed the dominant immune subset in these orthotopic tumors, with $\mathrm{Ly}_{6 \mathrm{G}}{ }^{+}$neutrophils comprising only a small percentage of the total $\mathrm{CD} 45^{+}$population (Fig. 2A). This recapitulated our findings in autochthonous TMPRSS2ERG ${ }^{\text {pce }}$ and TMPRSS2-ERG ${ }^{\text {pce- }}$ prostate tumors at both 6 and 12 months (Fig. S1A-S1B). In solid tumors the majority of TAMs differentiate from peripheral blood monocytes under the influence of colony stimulating factor-1 (CSF-1), with a smaller population of tissue-resident macrophages that persist through in situ proliferation (29-31). To determine the role of macrophages in CRPC, we 
treated mice bearing orthotopic PTE-82 tumors with an aCSF-1 neutralizing antibody for 7 days (Fig. 2B), which reduced the percentage of $\mathrm{CD} 11 \mathrm{~b}^{+} \mathrm{F} 480^{+} \mathrm{TAMs}$ without altering the frequency of other immune subsets (Fig. S2A). Another group of mice were treated with $0.6 \mathrm{mg} / \mathrm{mouse}$ Lupron Depot 16 days prior to tumor collection and processing for RNA sequencing to provide a comparison to standard-of-care therapeutics.

As shown in Fig. 2C, RNA sequencing followed by gene set enrichment analysis (GSEA) revealed that aCSF-1 treatment decreased the expression of genes related to steroid metabolism, bile acid metabolism and adipogenesis. Interestingly, these changes were very similar to those observed following treatment with Lupron (Fig. 2C, S2B). Depletion of TAMs elicited decreased expression of many AR-regulated genes (e.g. endogenous Tmprss2), along with a decrease in the global AR signature (normalized ES -1.3508) nearly equivalent to Lupron (normalized ES -1.4494) (Fig. 2D-S2C). As cholesterol is the main precursor of bile acids and the five major classes of steroid hormones, the results suggested that depletion of TAMs was causing a perturbation in cholesterol transport and/or metabolism. We reasoned that this perturbation could interfere with androgen biosynthesis in the tumor microenvironment, thereby explaining the reduced AR signature observed following TAM depletion. To evaluate this possibility, we utilized a LC-MS/MS metabolomics platform to quantify the level of intra-tumoral androgens in mouse tissues (Fig. S2D) (32). As shown in Fig. 2E, aCSF-1 treatment decreased the overall level of steroids, including the most potent endogenous androgen, DHT. Combined, these data indicate that macrophages regulate androgen biosynthesis in CRPC, potentially through a role in cholesterol transport/metabolism.

\section{Macrophages directly regulate AR nuclear translocation and resistance to enzalutamide}

The AR functions by binding steroid hormones in the cytoplasm and translocating to the nucleus, where, in conjugation with co-factors, it regulates expression of target genes by binding to specific DNA sequences known as androgen response elements (33). Based upon the reduction in intratumoral DHT and the AR gene signature that followed depletion of TAMs (Fig. 2D-E), we measured the amount of AR nuclear localization within tumors following administration of $\alpha \mathrm{CSF}-1$. Nuclear AR staining was prevalent in control tumors (Fig. 3A) and correlated with infiltration by F4/80+ macrophages (Fig. S3A). In contrast, the reduction in TAMs that followed administration of aCSF-1 coincided with a significant reduction in nuclear AR (Fig. 3A-B). This was not due to reduced AR protein expression in aCSF-1 treated tumors, but rather sequestration of AR in the cytosol. Notably, the reduction in AR nuclear translocation following TAM depletion was equivalent to treating mice with Lupron (Fig. 3A). In addition, upon analyzing autochthonous TMPRSS2-ERG ${ }^{\text {pce }}$ at 6 and 12 months, we found a positive correlation between nuclear localization of $A R$ and the extent of macrophage infiltration (Fig. S3B). Intriguingly, this correlation was not observed in autochthonous 
TMPRSS2-ERG ${ }^{\text {pce- }}$ tumors (Fig. S3B). Thus, TAMs regulate AR activation and nuclear translocation, at least in CRPC tumors expressing an ETS fusion gene.

We next sought to determine whether macrophages could directly induce AR nuclear translocation. We first established an in vitro system using a castration-sensitive cell line that was highly dependent upon AR signaling for growth, PTE-24 (Fig. 1I). PTE-24 cells were co-cultured with bone marrow-derived macrophages (BMDMs) under androgen-deprived conditions (i.e., 5\% CSS) and AR nuclear localization was measured via confocal microscopy and quantified by the ratio of nuclear to cytoplasmic AR on a cell-by-cell basis (Fig. 3C). As shown in Fig. 3D-E, the addition of BMDMs induced a significant increase in AR nuclear localization. As a result of this androgeninduced AR activation, BMDMs promoted the proliferation of PTE-24 cells in androgen-deprived conditions and protected against the cytotoxicity of the AR inhibitor enzalutamide (Fig. 3F). AR nuclear localization was also enhanced during co-culture of BMDMs with the PT-09 and PTE-82 CRPC cell lines (Fig. S3C); however, these lines are resistant to enzalutamide (Fig. 1I) and coculture with BMDMs had only a minor impact on proliferation by PTE-82 cells (Fig. S3D).

\section{Macrophages regulate cholesterol bioavailability}

We were intrigued by our comparative transcriptomic studies showing downregulation of bile acidand steroid biosynthesis-related genes in tumors isolated from aCSF1 treated mice, which suggested altered cholesterol metabolism. We thus performed correlation analysis between macrophage-related genes (CSF1R, CD68, CD206) and genes involved in bile acid and lipid metabolism in TCGA data (Prostate Adenocarcinoma dataset, Cell 2015)(34). We observed that macrophage-related genes correlated with those related to cholesterol transport, such as ABCA1, ABCA8, ABCA6 (Fig. 4A). Since cellular cholesterol is maintained by a balance between uptake and synthesis, we queried whether de novo cholesterol synthesis might be reduced in the presence of high macrophage infiltrate, and observed an inverse correlation between macrophage markers and de novo cholesterol synthesis-related genes (ACAT2, HMGCR, HMGCS1, SQLE) in two prostate cancer patient datasets (Fig. 4B, S4A).

Cholesterol is mostly stored in the form of cholesteryl esters in intracellular lipid droplets. Accordingly, we quantified the lipid content of immune cells within tumors and found that TAMs had the highest level of lipid content, both in orthotopic and autochthonous TMPRSS2-ERG ${ }^{\text {pce }}$ tumors $^{-}$ (Fig. 4C, S4B-C). BMDMs also expressed numerous genes related to cholesterol influx/efflux at higher levels than the prostate cancer cell lines, including Abca1, Abcg1, Cd36, Lpl and Scarb1 (Fig. 4D). To evaluate if macrophages were capable of transferring cholesterol to tumor cells, we loaded RAW264.7 cells, a murine macrophage cell line, with pHrodo-Red conjugated LDL as a source of cholesterol (and associated fatty acids and proteins). Note that pHrodo is fluorescent only at an acidic $\mathrm{pH}$, signifying intracellular uptake via endocytosis and/or phagocytosis has occurred (Fig. 4E). Pre- 
loaded RAW264.7 cells were then co-cultured with the prostate cancer cell lines for 24 hours, and depending on the tumor cell line, up to $40 \%$ of the cancer cells acquired derivate components of LDL (Fig. 4E).

To evaluate whether prostate cancer cells were promoting cholesterol efflux by macrophages, we labeled cells with cholera toxin subunit B (CTB), which is capable of binding to cholesterol-rich lipid rafts in the plasma membrane. As shown in Fig. 4F, co-culture with cancer cells largely eliminated CTB labeling of RAW264.7 cells, demonstrating a reduction in plasma membrane cholesterol. Finally, we sought to determine if the presence of macrophages could promote the acquisition of cholesterol (or other LDL components) by adding pHrodo-Red conjugated LDL to either prostate cancer cells alone, or those in co-culture with RAW264.7 cells (Fig. 4G). RAW264.7 cells rapidly took up LDL and were mostly positive during the first image acquisition, but fluorescent signal began to appear in the adherent prostate cancer cells within 2 hours. In contrast, no fluorescent signal was observed when prostate cancer cells were incubated alone (Fig. 4G). Overall, these data suggest that macrophages are a rich source of cholesterol, are capable of transferring cholesterol to prostate tumor cells, and can increase cholesterol bioavailability.

\section{Activating LXR $\beta$ inhibits macrophage-mediated AR translocation in tumor cells.}

Our cancer cell lines expressed detectable, albeit low, levels of the first two enzymes required for the conversion of cholesterol into androgens (Cyp11a, Cyp17a), whereas we were unable to detect gene expression of the enzyme responsible for cholesterol side-chain cleavage (Cyp11a) in BMDMs (Fig. S5A). We therefore sought to determine if preventing cholesterol transfer between macrophages and tumor cells could restrict AR nuclear translocation as a surrogate of androgen production. MetaCore analysis of our RNAseq data revealed that tumors with high macrophage infiltration (i.e. IgG control) were enriched in a Liver X Receptor (LXR)-regulated transcriptional program including target genes such as Abca1, Abcg1, Scarb1 and Cd36. Macrophages are generally thought to unload cholesterol via efflux mediated via the ATP-binding cassette transporters A1 and G1 (ABCA1 and ABCG1) to apolipoprotein A1 and high-density lipoproteins (HDLs), respectively, a process known to regulate atherosclerosis (35-37). Macrophage expression of these transporters has also recently been shown to promote the growth of ovarian cancer (38). We therefore crossed $A b c a 1^{f / f l} / A b c g 1^{f / f l}$ mice with LysM-Cre ${ }^{+}$mice to generate myeloid-specific loss of ABCA1 and ABCG1. Surprisingly, the extent of AR nuclear positivity within orthotopic tumors was equivalent in $L y s M$-cre ${ }^{-}$and $L y s M-$ cre $^{+}$ animals (Fig. 5A). There was also no difference in the growth of orthotopic tumors between the two genotypes (Fig. 5B). Consistent with this, BMDMs generated from $L y s M-C r e^{-}$and $L y s M-C r e^{+}$mice were equally capable of inducing AR nuclear translocation (Fig. 5C). AR nuclear translocation in vitro was similarly unchanged by a blocking antibody against CD36 or the addition of a specific scavenger receptor class B type I (SR-B1, encoded by Scarb1) inhibitor, BLT-1 (Fig. S5B). 
It has recently been shown that macrophages have the ability to transfer cholesterol through direct transcellular cholesterol movement in an ABCA1/G1-independent manner (39). Although the molecules involved in this process are unknown, upon re-examining our live imaging data we noticed that pHrodo-Red fluorescence in cancer cells occurred within those in proximity to RAW264.7 cells (Fig. 4E). We therefore sought to determine if inhibiting cholesterol influx and/or inducing cholesterol efflux in tumor cells would restrict AR nuclear translocation by activating the LXR transcription factors, which function as the master regulators of cholesterol homeostasis. Both BMDMs and prostate cancer cells expressed detectable levels of the genes encoding LXRa (Nr1h3) and LXRß (Nr1h2); however, Nr1h2 was expressed at much higher levels than Nr1h3 in the prostate cancer cell lines (Fig. 5D). As expected, the LXRaß inverse agonist, SR943, downregulated expression of Abca1 in cancer cells, while an $L X R \beta$ agonist currently in clinical trials, RGX-104, had the reverse effect (Fig. S5C). RGX-104 was also able to functionally inhibit prostate cancer cells from taking up pHrodo-Red LDL during a 24-hour incubation (Fig. S5D). We therefore examined the ability of SR943 and RGX104 to inhibit AR nuclear translocation during BMDM and prostate cancer cell co-culture. As shown in Fig. 5E, SR943 only slightly reduced the extent of $A R$ in the nucleus, while RGX-104 completely prevented AR nuclear translocation induced by the presence of BMDMs. This was not due to a direct effect on basal AR activity as expression of the AR target gene, Fkbp5, was not impacted by either the inverse agonist or agonist when BMDMs were not present (Fig. S5E). Collectively, these data demonstrate that cholesterol exchange between macrophages and prostate tumor cells enhances AR activation.

\section{Macrophage depletion sensitizes CRPC to Lupron}

Based upon the ability of TAMs to regulate the level of intratumoral androgens (Fig. 2E), AR nuclear localization (Fig. 3A) and AR-dependent gene expression (Fig. 2D), we evaluated the clinical relevance of these findings within the Decipher GRID ${ }^{\mathrm{TM}}$ registry data of 635 prostatectomy tumor samples with whole transcriptome data. As macrophages and AR nuclear localization were only correlated in the TMPRSS2-ERG ${ }^{\text {pce+ }}$ murine model (Fig. S3A) we stratified patients by ETS status and compared the ADT response signature with expression of macrophage-associated genes (CD68, CD163, CSF1R, or MRC1). In ETS ${ }^{+}$tumors we found that the genomic ADT response signature was inversely correlated with the presence of macrophages, whereas this association was not observed in ETS- tumors (Fig. 6A). Accordingly, we evaluated if depleting TAMs could reduce tumor growth and/or enhance survival in the TMPRSS2-ERG+ orthotopic CRPC model system (Fig. 6B). Treatment with either aCSF1 or Lupron alone had only a small impact on the growth of tumors as measured by MRI; however, a more significant reduction in tumor growth was observed when these therapies were combined (Fig. 6C, S6A-B). We therefore extended these studies to evaluate survival as measured by tumor size $\left(<1200 \mathrm{~mm}^{3}\right)$, organ obstruction, or overall animal health. Over the course of three 
bioRxiv preprint doi: https://doi.org/10.1101/2021.03.24.436480; this version posted March 24, 2021. The copyright holder for this preprint (which was not certified by peer review) is the author/funder. All rights reserved. No reuse allowed without permission.

independent experiments we observed a significant improvement in survival in mice treated with aCSF1 and Lupron, but not in mice treated with either agent along (Fig. 6D). Thus, targeting TAMs can improve response to ADT in ETS ${ }^{+}$CRPC. 


\section{Discussion}

AR signaling remains active in CRPC and plays a critical role in disease progression, as evidenced by the success of enzalutamide and abiraterone in extending survival in the metastatic setting (6). Compensatory growth factors secreted by other cell types in the tumor microenvironment are also thought to promote castration resistance (40). Castrating tumor-bearing mice elicits an influx of leukocytes, including B cells, neutrophils, and macrophages into prostate tumors, which are able to drive the emergence of CRPC by regulating senescence and proliferation (41-45). These studies point towards a feed-forward signaling loop in which cell stress induces expression of chemokines that promote leukocyte accumulation and drive the growth and development of CRPC. Accordingly, reducing infiltration of macrophage and neutrophils through the use of a CSF1R or CXCR2 antagonist, respectively, enhances the efficacy of ADT $(42,44)$. Critically, increased macrophage infiltration has been described in radical prostatectomy samples from ADT-treated patients, and high macrophage infiltration is associated with increased risk of biochemical recurrence (46).

Despite the association between macrophages and CRPC, the mechanism by which TAMs regulate response to ADT is unclear. CD11 $\mathrm{b}^{+} \mathrm{Ly} 6 \mathrm{G}^{+}$myeloid cells in prostate tumors can act as an important source of IL-1 receptor antagonist and IL-23 $(44,46)$, whereas no specific macrophagederived factors have been shown to drive the development of CRPC. Here we describe how macrophages regulate local steroid biosynthesis via enhancing the uptake of extrinsic cholesterol by prostate cancer cells, thereby leading to AR signaling and resistance to lupron in a model of CRPC. De novo steroidogenesis begins with cholesterol translocation into the mitochondria where the ratelimiting enzyme CYP11A1 converts cholesterol to pregnenolone, followed by series of enzymatic reactions to produce various steroids such as androgens, estrogens or glucocorticoids (47). Recently, glucocorticoids produced by TAMs were shown to elicit $\mathrm{T}$ cell dysfunction and resistance to immunotherapy in the MC38 colon carcinoma model (11). However, another recent study reported little to no expression of Cyp11a1 in TAMs within B16-F10 melanoma tumors, instead noting high expression by $\mathrm{T}$ cells and granulocytes (48). In our study, we did not detect Cyp11a1 in BMDMs, pointing towards the transfer of cholesterol rather than pregnenolone or other downstream derivatives in vitro. Prostate cancer cells also express most steroidogenic enzymes and have the ability to convert cholesterol to androgens (49-51). However it remains possible that there are other mechanisms by which macrophages regulate steroidogenesis, as reported in early studies investigating modulation of Leydig cell steroidogenesis by testicular macrophages (52).

Lipid transporters and scavenger receptors have emerged as potential targets in various types of solid malignancies $(53,54)$. In the ID8 ovarian cancer model, genetic ablation of $A b c a 1 / A b c g 1$ in myeloid cells decreased tumor growth, although whether this was through altered macrophage function or reduced cholesterol transfer to tumor cells is unclear (38). Similar to this study, we saw that the presence of tumor cells induced cholesterol efflux in macrophages. However, using the same 
genetic system we did not observe changes in prostate tumor growth or the ability of macrophages to promote AR nuclear localization in vitro or in vivo. Indeed, we found that macrophages can directly transfer cholesterol in serum free medium lacking lipoproteins, which would limit the ability of ABCA1/G1 to transfer cholesterol onto HDL particles. Inhibition of the HDL receptor SR-B1 also had minimal impact on the ability of BMDMs to promote AR nuclear localization in vitro. Our results are therefore in line with a recent a study that reported transcellular transfer of macrophage cholesterol to smooth muscle cells in the absence of serum or HDL (55). Additional work is required to delineate the molecular mechanism by which this transfer occurs.

In addition to PTEN and TP53 alterations, approximately $40 \%$ of prostate cancers carry recurrent rearrangements of the ETS family of transcription factors, of which fusion of ERG to the membrane protease TMPRSS2 represents the most common (56). Murine studies have shown that the TMPRSS2-ERG fusion increases early onset invasive prostate cancer through changes in the AR cistrome and enhancing AR output (28). TMPRSS2-ERG gene fusion status is also associated with a high androgen-regulated gene expression in early onset prostate cancer $(57,58)$. Apart of the transcriptional impact, TMPRSS2-ERG ${ }^{+}$patients have androgen profiles that differ substantially from TMPRSS2-ERG- patients, including increased DHT/testosterone ratios (59). These data are consistent with our preclinical observations regarding the correlation between macrophages and $A R$ nuclear translocation in the autochthonous and orthotopic TMPRSS2-ERG ${ }^{+}$murine model, and with the inverse correlation between macrophage infiltration and the genomic ADT response signature in $\mathrm{ETS}^{+}$patients. Cumulatively, these results suggest that macrophage-targeted agents such as CSF1R inhibitors should be directed towards patients with ETS ${ }^{+}$prostate cancer.

Collectively, our data show that macrophages deliver cholesterol to prostate tumor cells as an example of metabolic cooperation, thereby increasing intratumoral androgen production and activation of the AR. Whether targeting macrophages, cholesterol transfer, or cholesterol metabolism will prove therapeutically viable in prostate cancer remains to be determined. Interestingly, $L X R \beta$ agonists induce apoptosis of immunosuppressive myeloid cells through enhanced expression of ApoE, thereby driving $T$ cell responses in melanoma (60-62). Combined with their ability to restrict the cholesterol pool required for tumoral androgen synthesis (51), LXR $\beta$ agonists could therefore have profound impacts on the tumor microenvironment and offer unique opportunities for combinatorial therapies. Regardless of the approach taken, it is likely that macrophage-targeted therapies will display limited efficacy as single agents and that combinatorial approaches utilizing ADT should be evaluated clinically. 
Acknowledgements: The authors would like to thank Noel Clark, Sean Yoder, Antonio Ortiz, Jayden Cline and Joseph Johnson for technical assistance. The authors also thank John Cleveland, Conor Lynch, Jingsong Zhang and Paulo Rodriguez for scientific discussion.

Author Contributions: Conceptualization, A.E. and B.R.; Methodology, A.E. and M.L.; Formal Analysis, A.E., S.A., J.D., X.Y.; Investigation, A.E., W.D.V., M.L., A.K., K.S., L.N., Writing - Original Draft, A.E. and B.R.; Writing - Review \& Editing, A.E., K.Y., X.Y., J.K., R.A.G, and B.R.; Visualization, A.E. and B.R.; Supervision, R.G., K.Y., J.K., R.A.G., and B.R; Funding Acquisition, R.A.G. and B.R.

Financial Support: R.A.G is supported by the NIH/NCI (U54CA193489) and the V Foundation. B.R. is supported by the $\mathrm{NIH} / \mathrm{NCI}$ (R00CA185325, R01CA230610) and internal funding from Moffitt Cancer Center. This work was supported by Moffitt Cancer Center Proteomics and Metabolomics, Small Animal Imaging Lab, Flow Cytometry, Molecular Genomics, Analytic Microscopy, and Tissue Core Facilities, all comprehensive cancer center facilities designated by the National Cancer Institute (P30CA076292).

Conflict of Interests: A.E., J.K. and B.R. have courtesy faculty appointments at the University of South Florida, Tampa, FL 33620. A.E. has a faculty appointment at Mansoura University, Mansoura Egypt, 35516. B.R. has received payments from Merck \& Co., Inc. and Roche Farma S.A. for consulting, and has had sponsored research agreements with TESARO: A GSK Company. The other authors declare no potential conflicts of interest. 


\section{Figure Legends}

Figure 1. Development of syngeneic mouse models representing CRPC. A) Mouse model

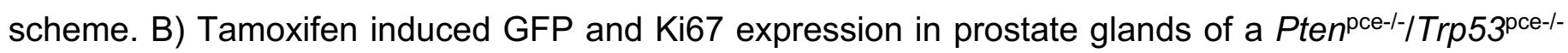
ITMPRSS2-ERG ${ }^{\text {pce+ }}$ mouse after 2 month of tamoxifen administration, $\mathrm{n}=1$. C) Detection of prostate

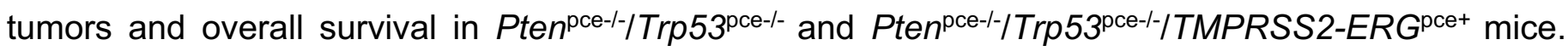
Days reflect time post tamoxifen administration. Tumor incidence and size were monitored by monthly MRI starting at 5-6 months. $n=17-19$ mice, pooled from two independent cohorts. Significance between the two groups was determined by log-rank. D) Hematoxylin and eosin (H\&E) and Masson's trichrome staining in Pten ${ }^{\text {pce-l-lTrp53 }}$ were harvested from mice at 6 and 12 months of age. E) Representative T2-weighted image by MRI

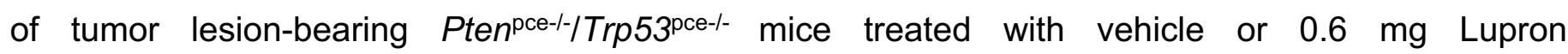
subcutaneously every 28 days for two cycles, starting 4 months after tamoxifen administration. $n=3-5$ mice per group, data from a one cohort of mice. F) Expression of Krt5, Krt8, Krt14, Krt18 in the

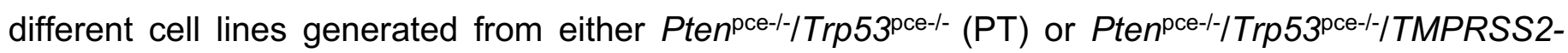
$E R G^{\text {pce+ }}(\mathrm{PTE})$ mice at end-stage. $\mathrm{n}=6$ from one experiment, with data shown as the mean $\pm \mathrm{SEM}$. TRAMP-C2 cell line was used as a comparison. Representative confocal microscopy immunofluorescent images of the PTE-82 cell line stained for cytokeratin 14 and cytokeratin 18 are shown to the right. Similar results were obtained for the PT-09, PT-25, and PTE-24 lines. Images represent one of two independent experiments. G) Expression of $A r$ in the different cell lines. $n=3$ from one experiment, data shown as the mean \pm SEM. H) Confocal microscopy immunofluorescent images of the PTE-82 cell line either treated with $10 \mathrm{nM}$ DHT or left untreated in charcoal stripped serum (CSS). Androgen receptor (red) and DAPI (blue). Images are representative of one of three independent experiments. Dose response curve of enzalutamide in 5 different prostate cancer cell lines. Phase contrast images were acquired at $8 \mathrm{hr}$ intervals using Incucyte, with confluence per well calculated per cell line. $n=3$, data shown as the mean \pm SEM from one of at least three independent experiments.

Figure 2. Macrophages are associated with cholesterol transport and steroid hallmarks. A) Immune infiltration in orthotopic models of prostate cancer, as determined by flow cytometry. $\mathrm{n}=5$ mice per group, with results from one of three independent experiments shown as the mean. B) Treatment schematic for Lupron and a-CSF1. C) Gene set enrichment analysis (GSEA) following whole tumor RNA sequencing. The normalized enrichment score is shown comparing tumors from mice treated with $\alpha$-CSF1 versus an $\lg G$ control. Data reflects 3 tumors per group from a single experiment. D) Enrichment plots of the Androgen Response Hallmark show for tumors from mice treated with a-CSF1 or Lupron, compared to the IgG control. E) LC-MS/MS quantification of pregnenolone, progesterone, androstenedione, DHEA, 5-androstenediol, testosterone and DHT in 
fresh tumor tissues. $n=5-6$ mice per group, from one of two independent experiments. Measurements were normalized by tissue weight. Significance determined by unpaired t-test for DHT. * $p<0.05$.

Figure 3. Macrophages directly regulate AR nuclear translocation. A) Representative immunohistochemistry for tumor macrophages (F4/80) and AR within orthotopic prostate tumors from mice treated with $\operatorname{lgG}$ (control), a-CSF or Lupron. B) Quantification of the percent of F4/80 cells and $\mathrm{AR}^{+}$nuclei in serial sections. $\mathrm{n}=5-6$ mice per group from one experiment. 4-5 regions of interest (ROI) per each slide were selected in non-necrotic areas of the tumor. Significance determined by unpaired t-test and shown as ${ }^{* *} p<0.001$. C) Experimental schematic for cancer cell and BMDM co-culture and quantification of nuclear AR by confocal microscopy. D) Kernel density estimation of the AR nuclear to cytoplasmic ratio on a cell-by-cell basis following incubation of PTE-24 cancer cell line alone, or in co-culture with BMDMs under androgen-deprived conditions (i.e., charcoal-stripped serum, CSS) for 72 hrs. $n=3$, data from one of at least three independent experiments. Significance determined by Mann-Whitney. E) Representative confocal microscopy images of the GFP ${ }^{+}$PTE-24 cell line. AR (red), F4/80 (white) and DAPI (blue) fluorescence is shown. F) Impact of BMDMs on the proliferation of PTE-24 cells in the presence of serial concentrations of enzalutamide. Cell proliferation was monitored using live imaging with phase contrast images acquired every $6 \mathrm{hr}$. Data shown as the mean \pm SEM and reflects one of 2 independent experiments. Significance determined by two-way ANOVA.

Figure 4. Macrophages transfer cholesterol to prostate cancer cells. A) Intergene correlation analysis of genes associated with macrophage infiltration (CD86, CSF1R, MRC1, CD163) and bile acid metabolism from TCGA. B) Intergene correlation analysis of genes associated with macrophage infiltration and de novo cholesterol synthesis (ACAT1/2, HMGCR, HMGCS1, SQLE). Analysis was performed with the Corrplot package in $\mathrm{R}$. Correlation coefficients are indicated by changes in circle color and size. C) Neutral lipid staining in immune cells within orthotopic PTE-82 tumors, as measured by BODIPY-493/503 staining in single cell suspensions. $n=5$ mice, data shown as the mean \pm SEM from one of three independent experiments. D) Expression of cholesterol metabolismrelated genes in prostate cancer cell lines and BMDMs. $n=3$, data from one of three independent experiments. E) RAW264.7 cells were loaded with human LDL covalently conjugated to pHrodo Red for $3 \mathrm{hrs}$, then co-cultured with prostate cancer cells for $24 \mathrm{hrs}$ prior to flow cytometric analysis. Cells were cultured separately and then admixed just prior to data acquisition for the 0 min control. The acquisition of fluorescence by the CD45- cancer cells is highlighted in the $3^{\text {rd }}$ quadrant in red. Quantification of percent LDL-positive tumor cells is shown to the right. $n=3$ biological replicates from one of two independent experiments. Data are presented as mean \pm SEM. Student's $t$ test was utilized for statistical analysis and is shown as ${ }^{* * *} p<0.001$. F) Immunofluorescence staining of 
cholesterol rich lipid rafts in the plasma membrane of RAW264.7 cells using Cholera toxin B (red). Cells were cultured alone or with PTE-82 cells in CSS for 48 hrs. Only nuclear GFP- RAW264.7 cells are shown. $n=3$, data from one of two independent experiments. G) Live imaging of uptake of pHrodo Red-LDL $(0.5 \mu \mathrm{g} / \mathrm{ml})$ by PTE-82 tumor cells in the presence or absence of RAW264.7 cells. Arrows indicate tumor cells with red fluorescence. $n=3$, data reflects one of three independent experiments.

\section{Figure 5. Perturbing cholesterol homeostasis inhibits macrophage-mediated AR translocation.}

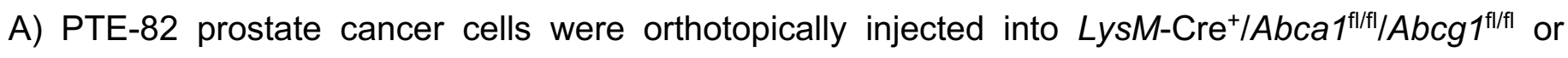
LysM-Cre-/Abca1 $1^{f / f l} / A b c g 1^{f / / f l}$ and nuclear expression of AR was evaluated after 19 days by IHC. Representative images are shown below. $\mathrm{n}=5-6$ mice per group, data shown as the mean $\pm \mathrm{SEM}$. Significance was determined by unpaired $t$ test assuming Gaussian distribution and with Welch's correction. B) Tumor volume in mice from A, as measured by MRI. Representative T2-weighted MRI images are shown. C) Nuclear to cytoplasmic AR ratio of GFP ${ }^{+}$PTE-82 cells cultured in CSS, either alone or in the presence of BMDMs derived from LysM-Cre ${ }^{+} / A b c a 1^{f / / f l} / A b c g 1^{f / / f l}$ or $L y s M-C r e^{-}$ $\mid A b c a 1^{f / f \mid} / A b c g 1^{f / / f l}$ donors. 6-8 randomly selected images from two wells of the chamber slides were pooled for analysis. Data reflects one of three independent experiments. Significance was determined by Mann-Whitney. D) Expression of $\operatorname{Nr} 1 h 2(\mathrm{LXR} \beta)$ and $N r 1 h 3(\mathrm{LXR \alpha})$ in prostate cancer cell lines and BMDMs. $n=3$, data shown as the mean \pm SEM from one of two independent experiments. E) Nuclear to cytoplasmic AR ratio of $\mathrm{GFP}^{+}$PTE-82 cells cultured in CSS or co-cultured with BMDMs. $5 \mu \mathrm{M}$ SR9243 (LXRa/ $\beta$ inverse agonist) or $5 \mu \mathrm{M}$ RGX-104 (LXR $\beta$ agonist) were added to co-cultures as indicated. 6-8 randomly selected images from two wells of the chamber slides were pooled for analysis. Data reflects one of three independent experiments. Significance determined by MannWhitney.

Figure 6. Macrophage depletion sensitizes CRPC to Lupron. A) Expression of macrophageassociated genes was correlated with ADT response score, with stratification by ETS status. B) PTE82 tumor cells were implanted orthotopically into prostates of C57 mice, which were then treated with Lupron depot and/or aCSF-1 as indicated. Tumor volumes for individual mice are shown for day 21. $\mathrm{n}=13-15$ mice per group, data pooled from two independent experiments. Significance was determined by one-way ANOVA and is show compared to the IgG control group, unless otherwise indicated. C) Tumor volume in treatment groups as measured by MRI. D) Survival as determine by tumor volume or organ obstruction. $n=21-23$ mice, data pooled from three experiments. Significance was determined via log-rank. 


\section{References}

1. Attar RM, Takimoto $\mathrm{CH}$, Gottardis MM. Castration-Resistant Prostate Cancer: Locking Up the Molecular Escape Routes. Clinical Cancer Research 2009;15:3251-5

2. Karantanos T, Corn PG, Thompson TC. Prostate cancer progression after androgen deprivation therapy: mechanisms of castrate resistance and novel therapeutic approaches. Oncogene 2013;32:5501-11

3. Nussbaum N, George DJ, Abernethy AP, Dolan CM, Oestreicher N, Flanders S, et al. Patient experience in the treatment of metastatic castration-resistant prostate cancer: state of the science. Prostate Cancer Prostatic Dis 2016;19:111-21

4. Sharifi N. Minireview: Androgen metabolism in castration-resistant prostate cancer. Mol Endocrinol 2013;27:708-14

5. Sharifi N, McPhaul MJ, Auchus RJ. "Getting from here to there"--mechanisms and limitations to the activation of the androgen receptor in castration-resistant prostate cancer. Journal of investigative medicine : the official publication of the American Federation for Clinical Research 2010;58:938-44

6. Litwin MS, Tan HJ. The Diagnosis and Treatment of Prostate Cancer: A Review. JAMA 2017;317:2532-42

7. El-Kenawi A, Gatenbee C, Robertson-Tessi M, Bravo R, Dhillon J, Balagurunathan Y, et al. Acidity promotes tumour progression by altering macrophage phenotype in prostate cancer. British journal of cancer 2019;121:556-66

8. DeNardo DG, Ruffell B. Macrophages as regulators of tumour immunity and immunotherapy. Nat Rev Immunol 2019;19:369-82

9. Ruffell B, Coussens LM. Macrophages and Therapeutic Resistance in Cancer. Cancer cell 2015;27:462-72

10. El-Kenawi AE, El-Remessy $A B$. Angiogenesis inhibitors in cancer therapy: mechanistic perspective on classification and treatment rationales. Br J Pharmacol 2013;170:712-29

11. Acharya N, Madi A, Zhang H, Klapholz M, Escobar G, Dulberg S, et al. Endogenous Glucocorticoid Signaling Regulates CD8(+) T Cell Differentiation and Development of Dysfunction in the Tumor Microenvironment. Immunity 2020;53:658-71 e6

12. Birbach A, Casanova E, Schmid JA. A Probasin-MerCreMer BAC allows inducible recombination in the mouse prostate. Genesis 2009;47:757-64

13. Ruffell B, Chang-Strachan D, Chan V, Rosenbusch A, Ho CM, Pryer N, et al. Macrophage IL10 blocks CD8+ $\mathrm{T}$ cell-dependent responses to chemotherapy by suppressing IL-12 expression in intratumoral dendritic cells. Cancer cell 2014;26:623-37

14. Foster BA, Gingrich JR, Kwon ED, Madias C, Greenberg NM. Characterization of prostatic epithelial cell lines derived from transgenic adenocarcinoma of the mouse prostate (TRAMP) model. Cancer Res 1997;57:3325-30

15. Zhang $\mathrm{X}$, Goncalves $\mathrm{R}$, Mosser DM. The isolation and characterization of murine macrophages. Curr Protoc Immunol 2008; Chapter 14:Unit 141

16. Vereyken EJ, Heijnen PD, Baron W, de Vries EH, Dijkstra CD, Teunissen CE. Classically and alternatively activated bone marrow derived macrophages differ in cytoskeletal functions and migration towards specific CNS cell types. J Neuroinflammation 2011;8:58

17. Celik HT, Serdar MA, Abusoglu S, Sezer S, Ozdemir S, Ozdemir O, et al. Determination of Serum Testosterone Levels with Liquid Chromatography-Isotope Dilution Tandem Mass Spectrometry and Comparision with Other Immunoassays. Biochem Anal Biochem 2012;1

18. Weng Y, Xie F, Xu L, Zagorevski D, Spink DC, Ding X. Analysis of testosterone and dihydrotestosterone in mouse tissues by liquid chromatography-electrospray ionization-tandem mass spectrometry. Anal Biochem 2010;402:121-8

19. Martin M. Cutadapt removes adapter sequences from high-throughput sequencing reads. 2011 2011;17:3 \%J EMBnet.journal

20. Dobin A, Davis CA, Schlesinger F, Drenkow J, Zaleski C, Jha S, et al. STAR: ultrafast universal RNA-seq aligner. Bioinformatics 2013;29:15-21 
21. Anders S, Pyl PT, Huber W. HTSeq--a Python framework to work with high-throughput sequencing data. Bioinformatics 2015;31:166-9

22. Love MI, Huber W, Anders S. Moderated estimation of fold change and dispersion for RNAseq data with DESeq2. Genome Biology 2014;15:550

23. Subramanian A, Tamayo P, Mootha VK, Mukherjee S, Ebert BL, Gillette MA, et al. Gene set enrichment analysis: A knowledge-based approach for interpreting genome-wide expression profiles. Proceedings of the National Academy of Sciences 2005;102:15545-50

24. Ashburner M, Ball CA, Blake JA, Botstein D, Butler H, Cherry JM, et al. Gene Ontology: tool for the unification of biology. Nature Genetics 2000;25:25-9

25. Awasthi S, Berglund AE, Abraham-Miranda J, Rounbehler RJ, Kensler KH, Serna AN, et al. Comparative genomics reveals distinct immune-oncologic pathways in African American men with prostate cancer. Clin Cancer Res 2020

26. Karnes RJ, Sharma V, Choeurng V, Ashab HA, Erho N, Alshalalfa M, et al. Development and Validation of a Prostate Cancer Genomic Signature that Predicts Early ADT Treatment Response Following Radical Prostatectomy. Clin Cancer Res 2018;24:3908-16

27. Ahearn TU, Pettersson A, Ebot EM, Gerke T, Graff RE, Morais CL, et al. A Prospective Investigation of PTEN Loss and ERG Expression in Lethal Prostate Cancer. J Natl Cancer Inst 2016;108

28. Chen Y, Chi P, Rockowitz S, laquinta PJ, Shamu T, Shukla S, et al. ETS factors reprogram the androgen receptor cistrome and prime prostate tumorigenesis in response to PTEN loss. Nature medicine 2013;19:1023-9

29. Lee AW. The role of atypical protein kinase $C$ in CSF-1-dependent Erk activation and proliferation in myeloid progenitors and macrophages. PLoS One 2011;6:e25580

30. Zhu Y, Herndon JM, Sojka DK, Kim KW, Knolhoff BL, Zuo C, et al. Tissue-Resident Macrophages in Pancreatic Ductal Adenocarcinoma Originate from Embryonic Hematopoiesis and Promote Tumor Progression. Immunity 2017;47:323-38 e6

31. Stanley ER, Chitu V. CSF-1 receptor signaling in myeloid cells. Cold Spring Harb Perspect Biol 2014;6

32. Liu M, Cline J, Elkenawi A, Ruffell B, J. K. Analysis of Cholesterol and Androgens in Mouse Prostate Cells by LC-MS/MS: HESI versus APCl". 68th ASMS Conference on Mass Spectrometry 2020

33. Mills IG. Maintaining and reprogramming genomic androgen receptor activity in prostate cancer. Nat Rev Cancer 2014;14:187-98

34. Cancer Genome Atlas Research N. The Molecular Taxonomy of Primary Prostate Cancer. Cell 2015;163:1011-25

35. Westerterp M, Murphy AJ, Wang M, Pagler TA, Vengrenyuk Y, Kappus MS, et al. Deficiency of ATP-binding cassette transporters $A 1$ and $\mathrm{G} 1$ in macrophages increases inflammation and accelerates atherosclerosis in mice. Circ Res 2013;112:1456-65

36. Ramji DP. Growth hormone-releasing peptides, CD36, and stimulation of cholesterol efflux: cyclooxygenase-2 is the link. Cardiovascular Research 2009;83:419-20

37. Chistiakov DA, Bobryshev YV, Orekhov AN. Macrophage-mediated cholesterol handling in atherosclerosis. J Cell Mol Med 2016;20:17-28

38. Goossens P, Rodriguez-Vita J, Etzerodt A, Masse M, Rastoin O, Gouirand V, et al. Membrane Cholesterol Efflux Drives Tumor-Associated Macrophage Reprogramming and Tumor Progression. Cell Metab 2019;29:1376-89 e4

39. He C, Hu X, Weston TA, Jung RS, Sandhu J, Huang S, et al. Macrophages release plasma membrane-derived particles rich in accessible cholesterol. Proc Natl Acad Sci U S A 2018;115:E8499-E508

40. Karlou M, Tzelepi V, Efstathiou E. Therapeutic targeting of the prostate cancer microenvironment. Nat Rev Urol 2010;7:494-509

41. Xu J, Escamilla J, Mok S, David J, Priceman S, West B, et al. CSF1R signaling blockade stanches tumor-infiltrating myeloid cells and improves the efficacy of radiotherapy in prostate cancer. Cancer Res 2013;73:2782-94 
42. Escamilla J, Schokrpur S, Liu C, Priceman SJ, Moughon D, Jiang Z, et al. CSF1 receptor targeting in prostate cancer reverses macrophage-mediated resistance to androgen blockade therapy. Cancer research 2015;75:950-62

43. Ammirante M, Luo JL, Grivennikov S, Nedospasov S, Karin M. B-cell-derived lymphotoxin promotes castration-resistant prostate cancer. Nature 2010;464:302-5

44. Calcinotto A, Spataro C, Zagato E, Di Mitri D, Gil V, Crespo M, et al. IL-23 secreted by myeloid cells drives castration-resistant prostate cancer. Nature 2018;559:363-9

45. Di Mitri D, Toso A, Chen JJ, Sarti M, Pinton S, Jost TR, et al. Tumour-infiltrating Gr-1+ myeloid cells antagonize senescence in cancer. Nature 2014;515:134-7

46. Gannon PO, Poisson AO, Delvoye N, Lapointe R, Mes-Masson AM, Saad F. Characterization of the intra-prostatic immune cell infiltration in androgen-deprived prostate cancer patients. J Immunol Methods 2009;348:9-17

47. Miller WL, Auchus RJ. The molecular biology, biochemistry, and physiology of human steroidogenesis and its disorders. Endocr Rev 2011;32:81-151

48. Mahata B, Pramanik J, van der Weyden L, Polanski K, Kar G, Riedel A, et al. Tumors induce de novo steroid biosynthesis in T cells to evade immunity. Nat Commun 2020;11:3588

49. Yang Y, Bai Y, He Y, Zhao Y, Chen J, Ma L, et al. PTEN Loss Promotes Intratumoral Androgen Synthesis and Tumor Microenvironment Remodeling via Aberrant Activation of RUNX2 in Castration-Resistant Prostate Cancer. Clinical cancer research : an official journal of the American Association for Cancer Research 2018;24:834-46

50. Cai C, Chen S, Ng P, Bubley GJ, Nelson PS, Mostaghel EA, et al. Intratumoral de novo steroid synthesis activates androgen receptor in castration-resistant prostate cancer and is upregulated by treatment with CYP17A1 inhibitors. Cancer research 2011;71:6503-13

51. Leon CG, Locke JA, Adomat HH, Etinger SL, Twiddy AL, Neumann RD, et al. Alterations in cholesterol regulation contribute to the production of intratumoral androgens during progression to castration-resistant prostate cancer in a mouse xenograft model. Prostate 2010;70:390-400

52. Hales DB. Testicular macrophage modulation of Leydig cell steroidogenesis. J Reprod Immunol 2002;57:3-18

53. Watt MJ, Clark AK, Selth LA, Haynes VR, Lister N, Rebello R, et al. Suppressing fatty acid uptake has therapeutic effects in preclinical models of prostate cancer. Science Translational Medicine 2019;11:eaau5758

54. Traughber CA, Opoku E, Brubaker G, Major J, Lu H, Lorkowski SW, et al. Uptake of highdensity lipoprotein by scavenger receptor class $B$ type 1 is associated with prostate cancer proliferation and tumor progression in mice. Journal of Biological Chemistry 2020;295:8252-61

55. He C, Jiang H, Song W, Riezman H, Tontonoz P, Weston TA, et al. Cultured macrophages transfer surplus cholesterol into adjacent cells in the absence of serum or high-density lipoproteins. Proceedings of the National Academy of Sciences 2020;117:10476-83

56. Tomlins SA, Rhodes DR, Perner S, Dhanasekaran SM, Mehra R, Sun XW, et al. Recurrent fusion of TMPRSS2 and ETS transcription factor genes in prostate cancer. Science 2005;310:644-8

57. Weischenfeldt J, Simon R, Feuerbach L, Schlangen K, Weichenhan D, Minner S, et al. Integrative genomic analyses reveal an androgen-driven somatic alteration landscape in earlyonset prostate cancer. Cancer Cell 2013;23:159-70

58. Gerhauser C, Favero F, Risch T, Simon R, Feuerbach L, Assenov Y, et al. Molecular Evolution of Early-Onset Prostate Cancer Identifies Molecular Risk Markers and Clinical Trajectories. Cancer Cell 2018;34:996-1011 e8

59. Knuuttila M, Mehmood A, Maki-Jouppila J, Ryberg H, Taimen P, Knaapila J, et al. Intratumoral androgen levels are linked to TMPRSS2-ERG fusion in prostate cancer. Endocr Relat Cancer 2018;25:807-19

60. Flaveny CA, Griffett K, El-Gendy Bel D, Kazantzis M, Sengupta M, Amelio AL, et al. Broad Anti-tumor Activity of a Small Molecule that Selectively Targets the Warburg Effect and Lipogenesis. Cancer cell 2015;28:42-56 
bioRxiv preprint doi: https://doi.org/10.1101/2021.03.24.436480; this version posted March 24, 2021. The copyright holder for this preprint (which was not certified by peer review) is the author/funder. All rights reserved. No reuse allowed without permission.

61. Tavazoie MF, Pollack I, Tanqueco R, Ostendorf BN, Reis BS, Gonsalves FC, et al. LXR/ApoE Activation Restricts Innate Immune Suppression in Cancer. Cell 2018;172:825-40 e18

62. Wu G, Wang Q, Xu Y, Li J, Zhang H, Qi G, et al. Targeting the transcription factor receptor LXR to treat clear cell renal cell carcinoma: agonist or inverse agonist? Cell Death Dis 2019;10:416 
bioRxiv preprint doi: https://doi.org/10.1101/2021.03.24.436480; this version posted March 24, 2021. The copyright holder for this preprint (which was not certified by peer review) is the author/funder. All rights reserved. No reuse allowed without permission.

A

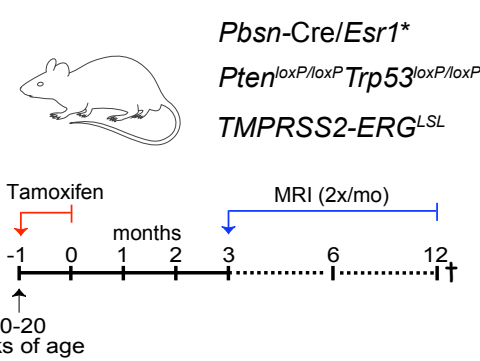

C
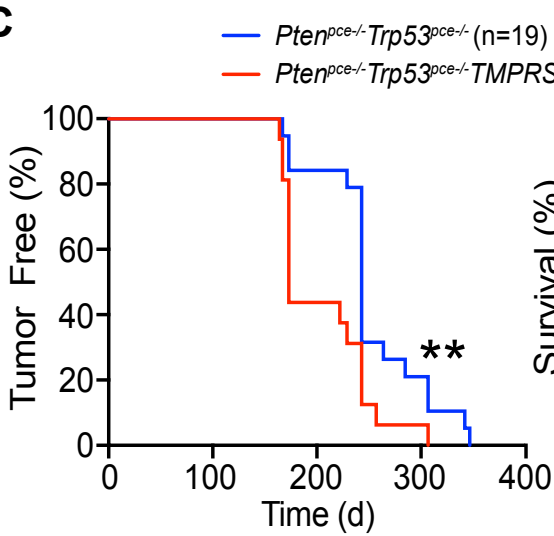

E

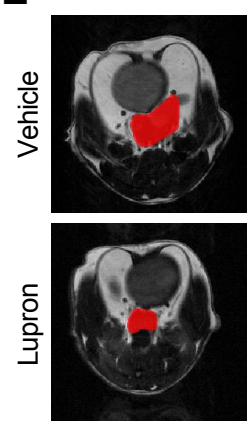

$\mathbf{F}$

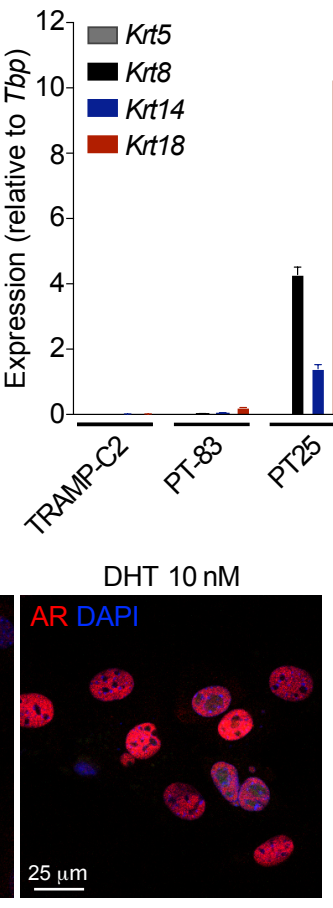

PTE-82

H
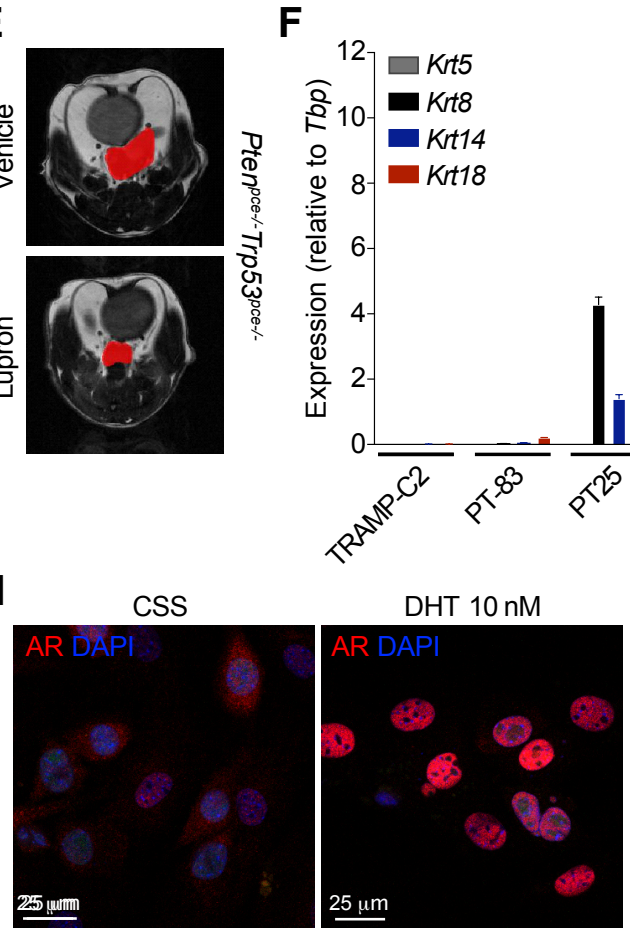

B

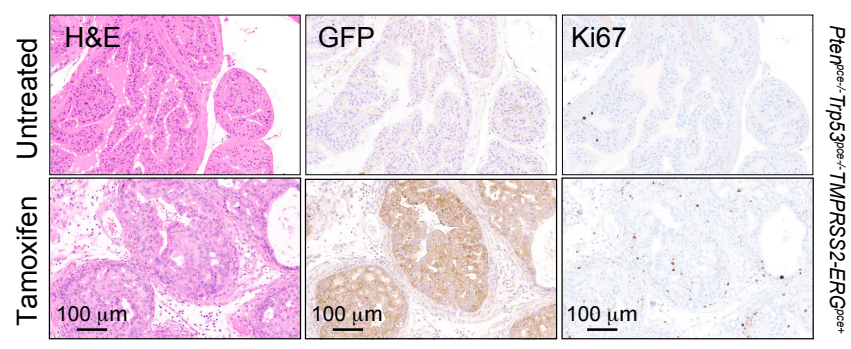

D Control 6 months 12 months

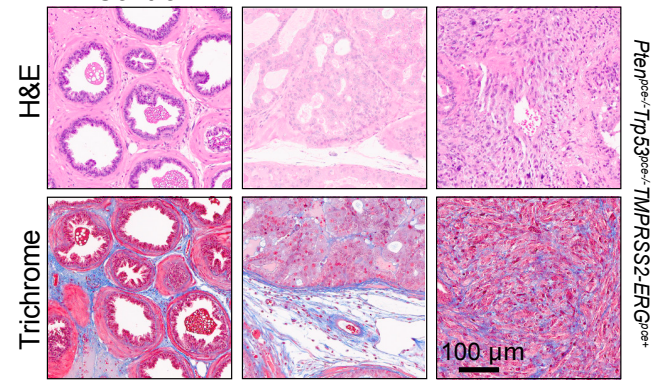

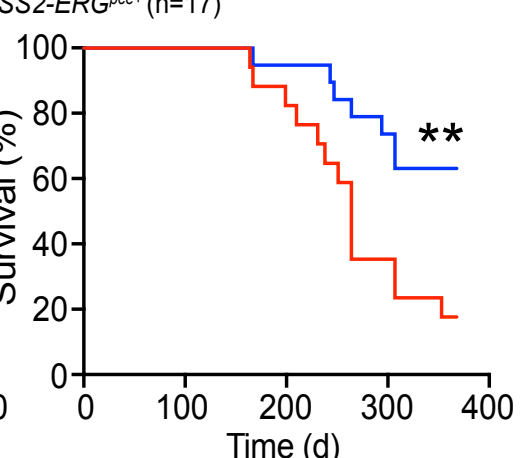

Time $(d)$

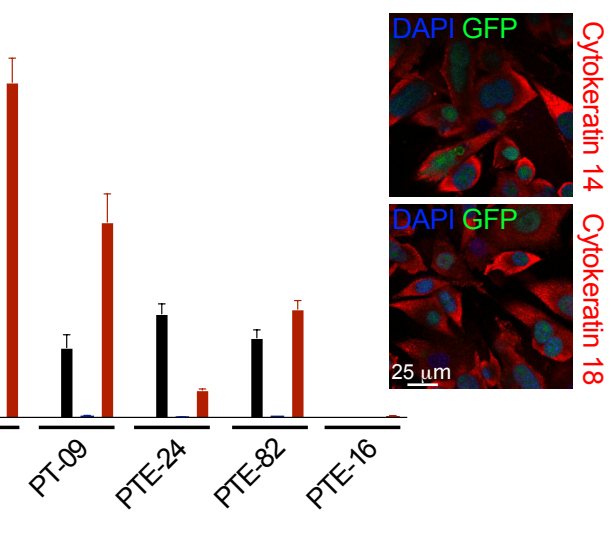

G

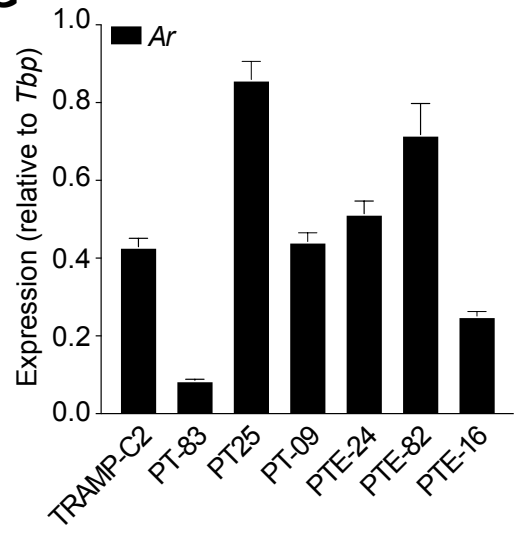


bioRxiv preprint doi: https://doi.org/10.1101/2021.03.24.436480; this version posted March 24, 2021. The copyright holder for this preprint (which was not certified by peer review) is the author/funder. All rights reserved. No reuse allowed without permission.

A

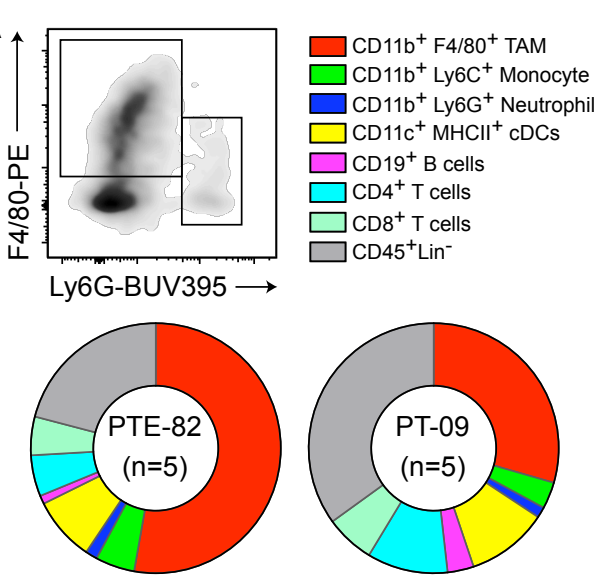

B

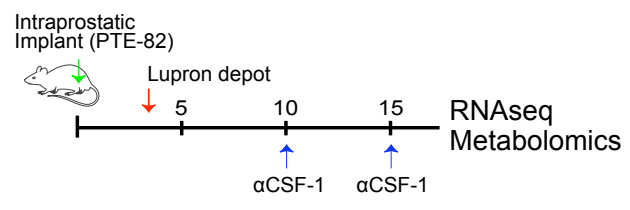

C

aCSF-1 vs. IgG

Normalized Enrichment Score

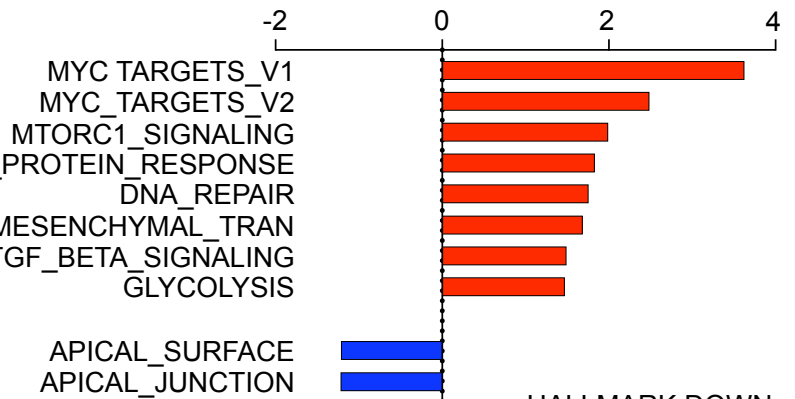

ANDROGEN RESPONSE $P \bar{E} R O X I S O M E$

FATTY_ACID_METABOLISM ESTROGEN_RESPONSE_EARLY ADIPOGEENESIS

XENOBIOTIC_METABOLISM ESTROGEN_RESTPONSE_LATE MYOGENEESIS

BILE_ACID_METABOLISM KRAS_SIGNALING_DN
D

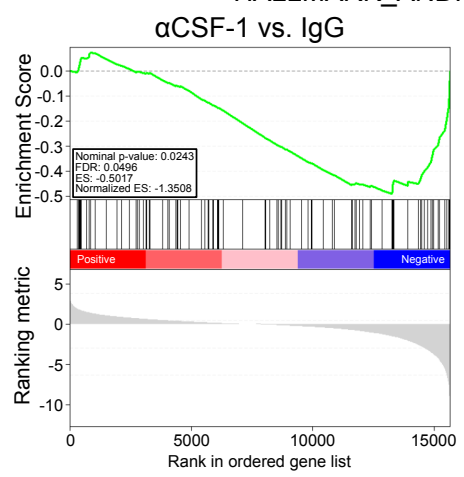

HALLMARK ANDROGEN RESPONSE

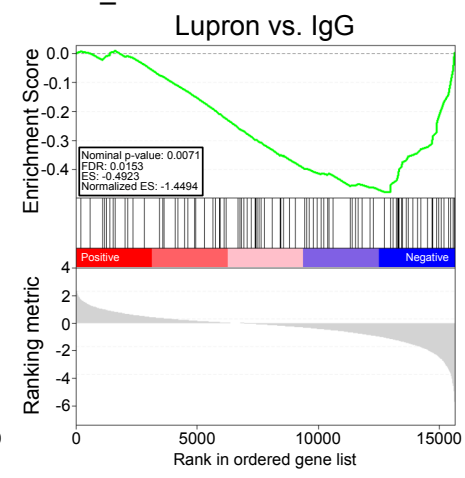

E

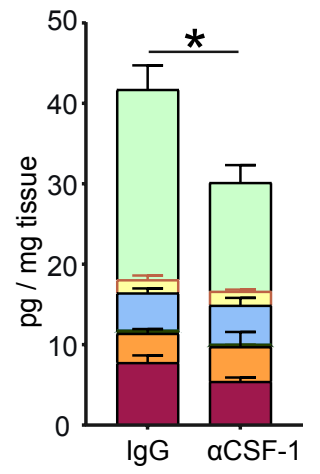

HALLMARK DOWN

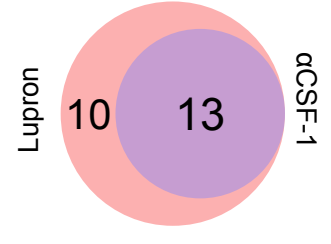

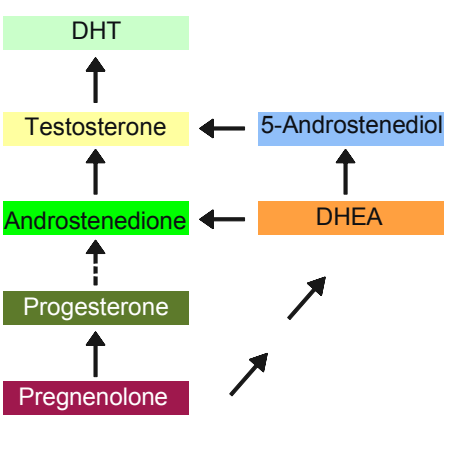


bioRxiv preprint doi: https://doi.org/10.1101/2021.03.24.436480; this version posted March 24, 2021. The copyright holder for this preprint (which was not certified by peer review) is the author/funder. All rights reserved. No reuse allowed without permission.

A

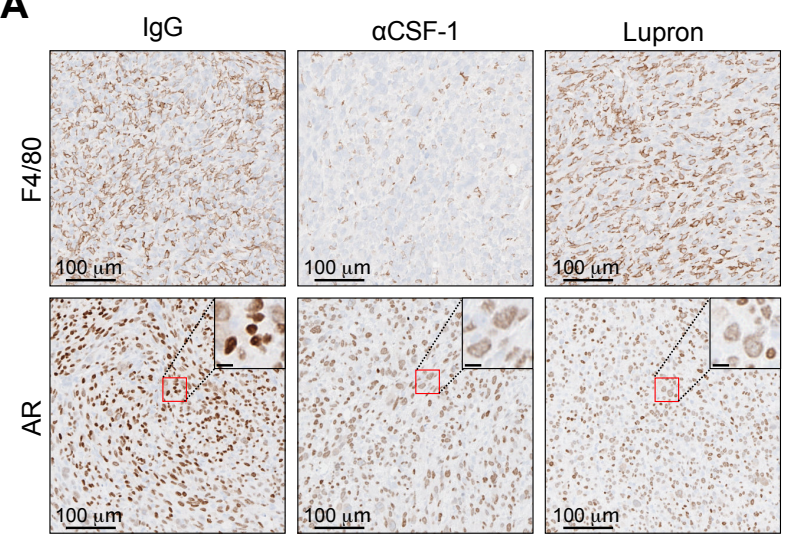

C

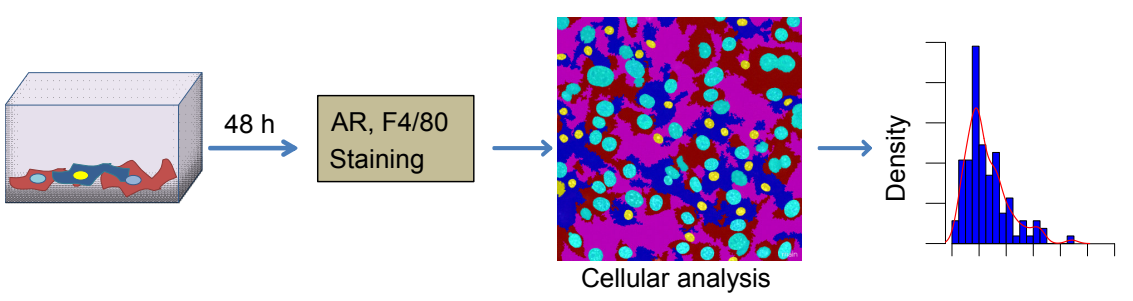

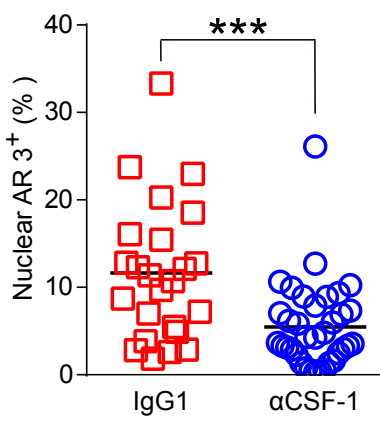

D

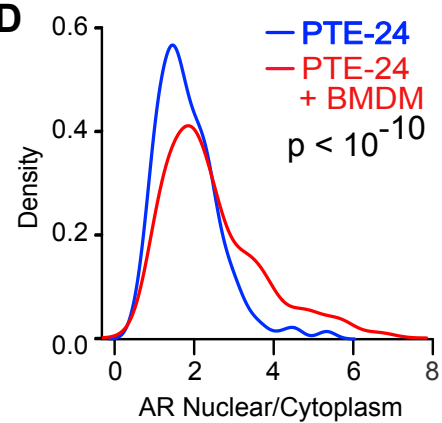

E

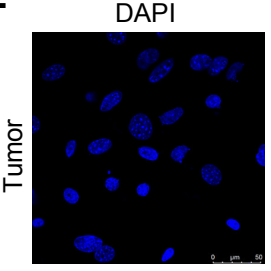

GFP
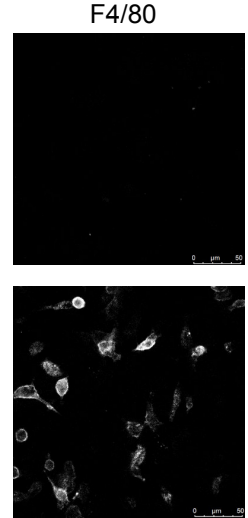

$10 \mu \mathrm{M}$ enzalutamide
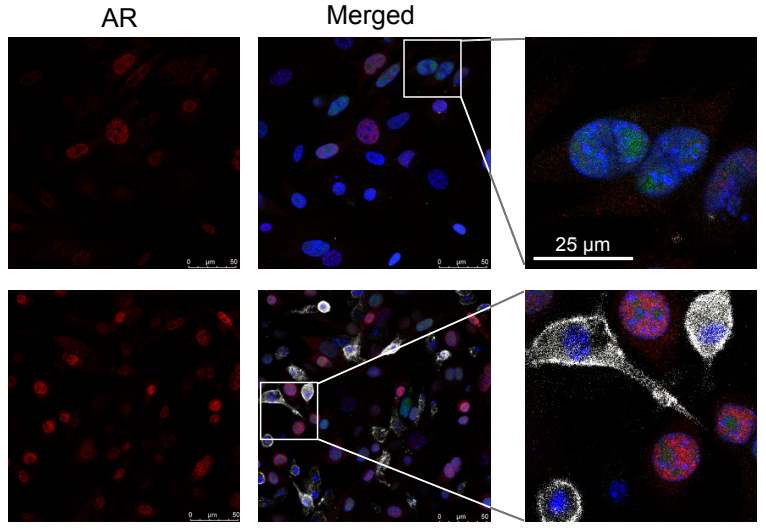

F
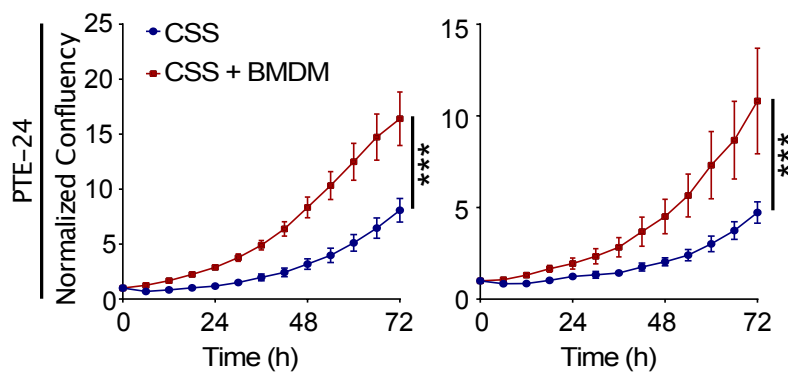

$20 \mu \mathrm{M}$ enzalutamide

$40 \mu \mathrm{M}$ enzalutamide
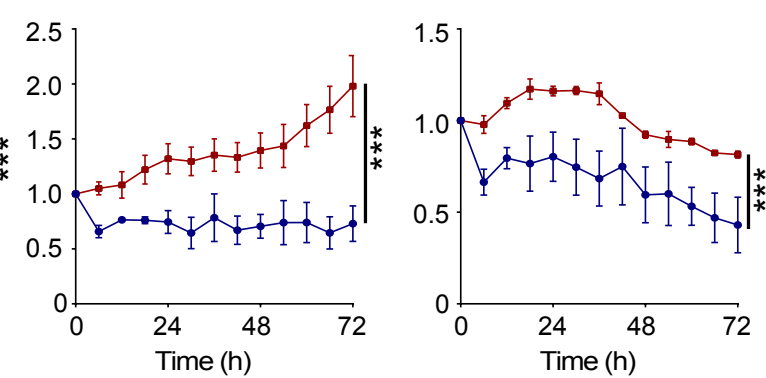
bioRxiv preprint doi: https://doi.org/10.1101/2021.03.24.436480; this version posted March 24, 2021. The copyright holder for this preprint (which was not certified by peer review) is the author/funder. All rights reserved. No reuse allowed without permission.

A

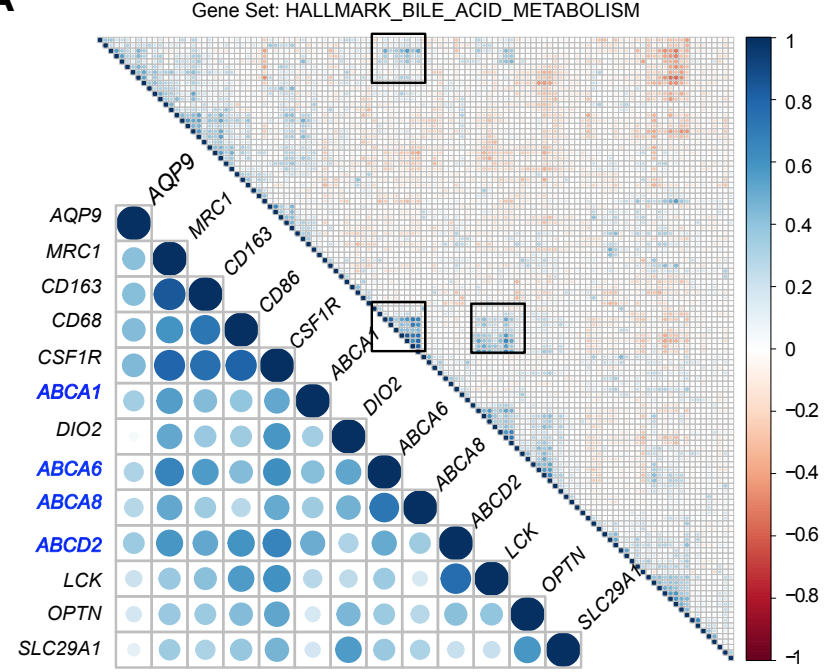

B

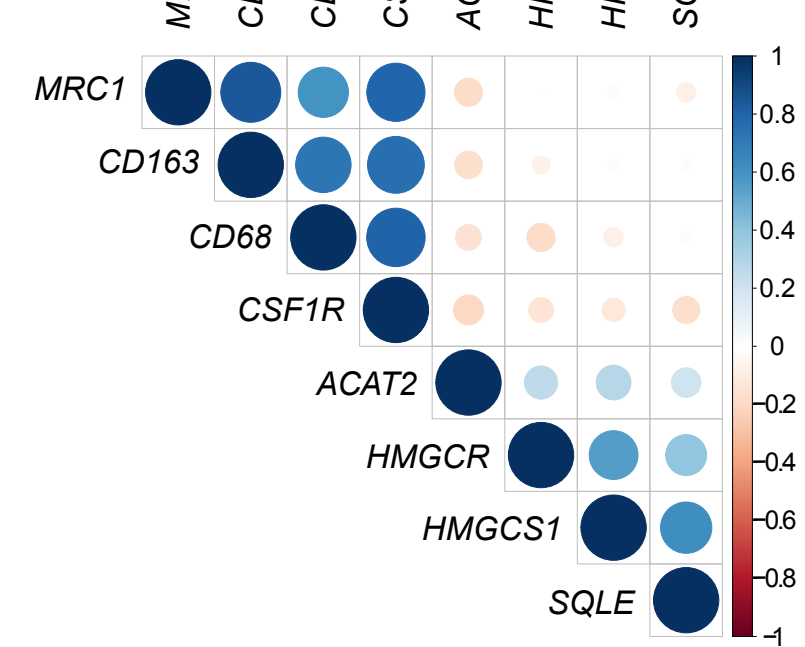

BMDM OPT-09 口PTE-24 $\Delta$ PTE-82

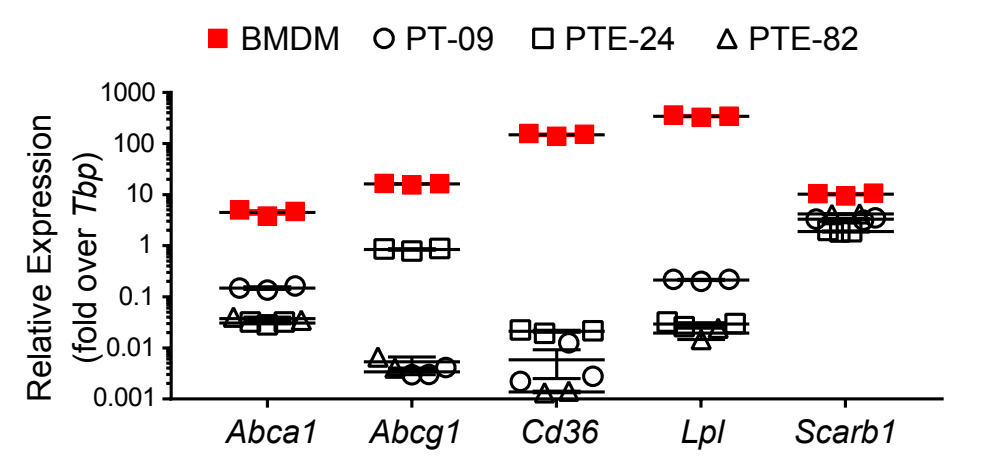

D
E

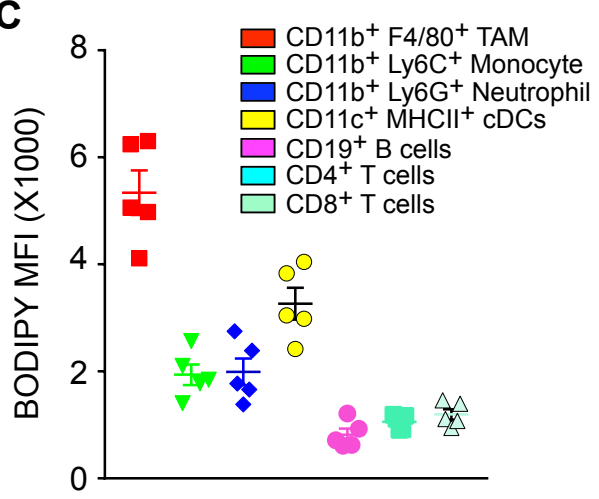

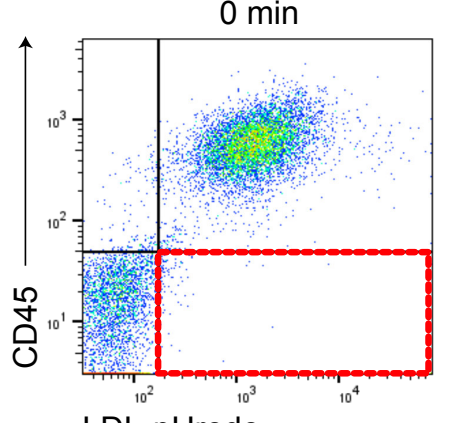

LDL-pHrodo

\section{$\mathbf{F}$}
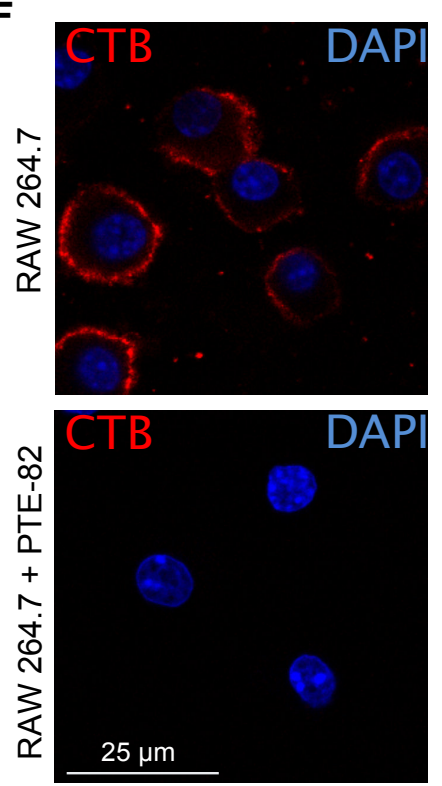
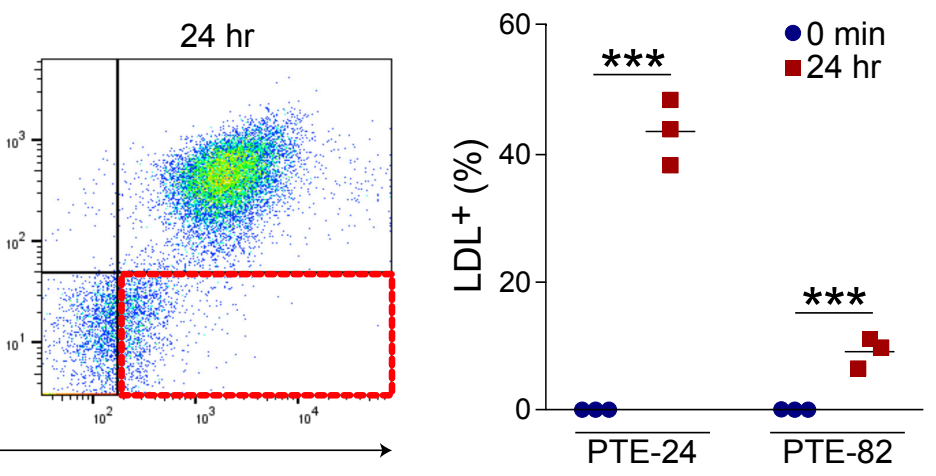

G
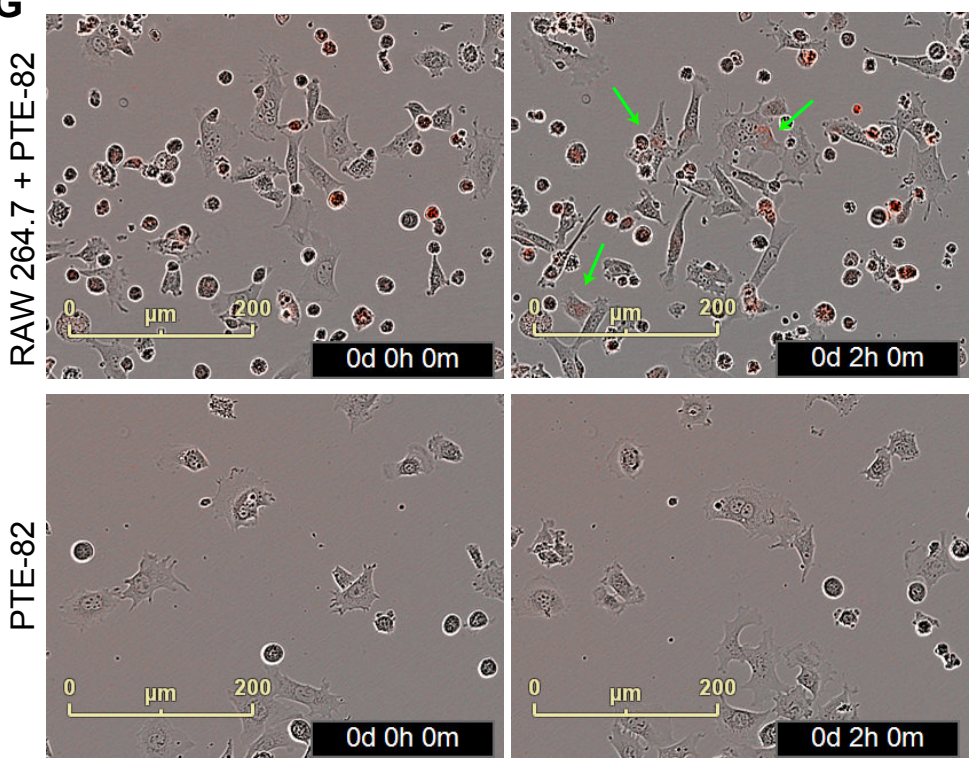
bioRxiv preprint doi: https://doi.org/10.1101/2021.03.24.436480; this version posted March 24, 2021. The copyright holder for this preprint (which was not certified by peer review) is the author/funder. All rights reserved. No reuse allowed without permission.

A
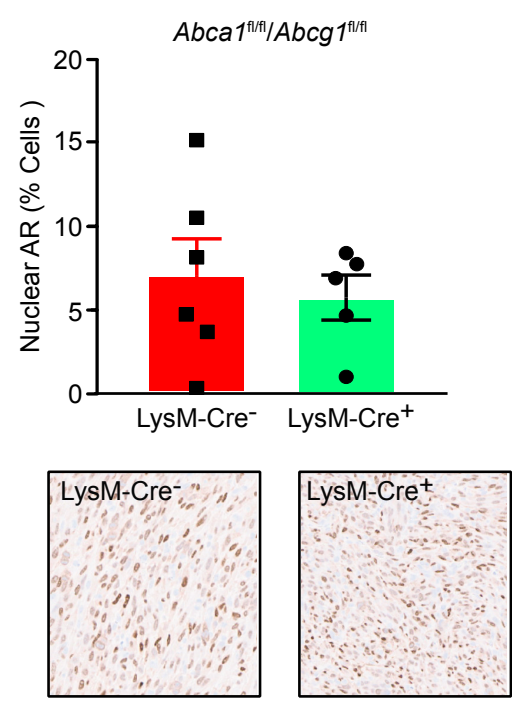

D

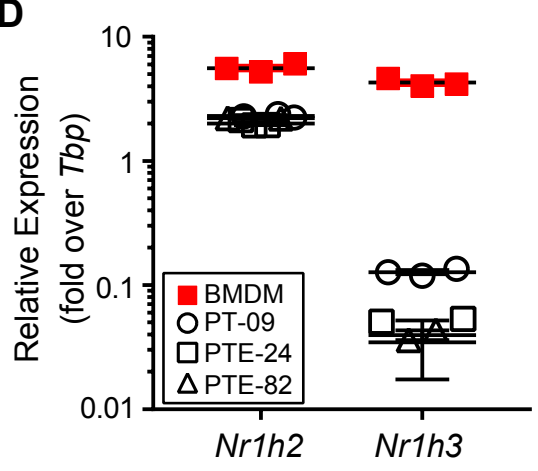

B
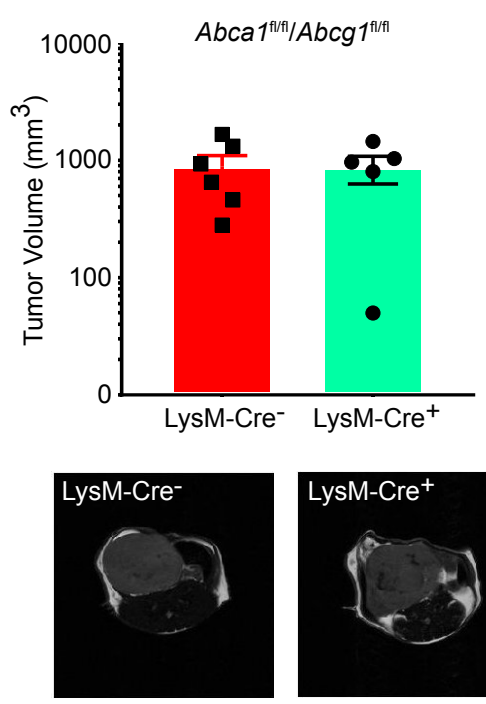

E

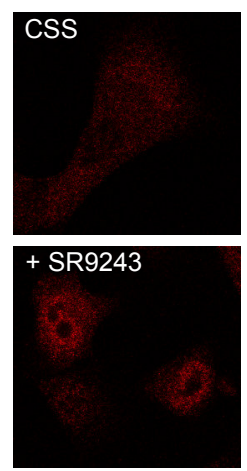

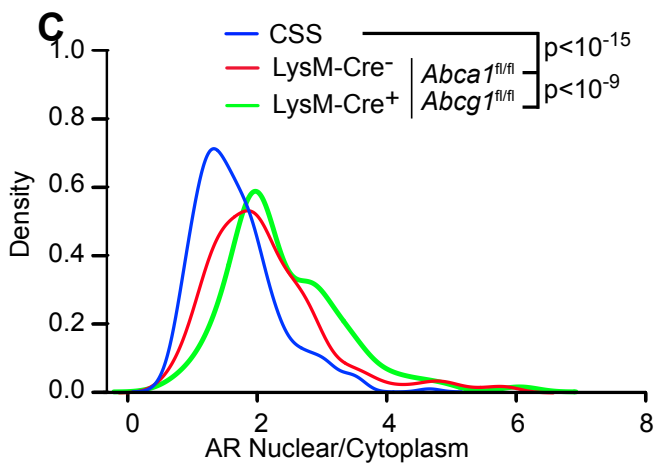
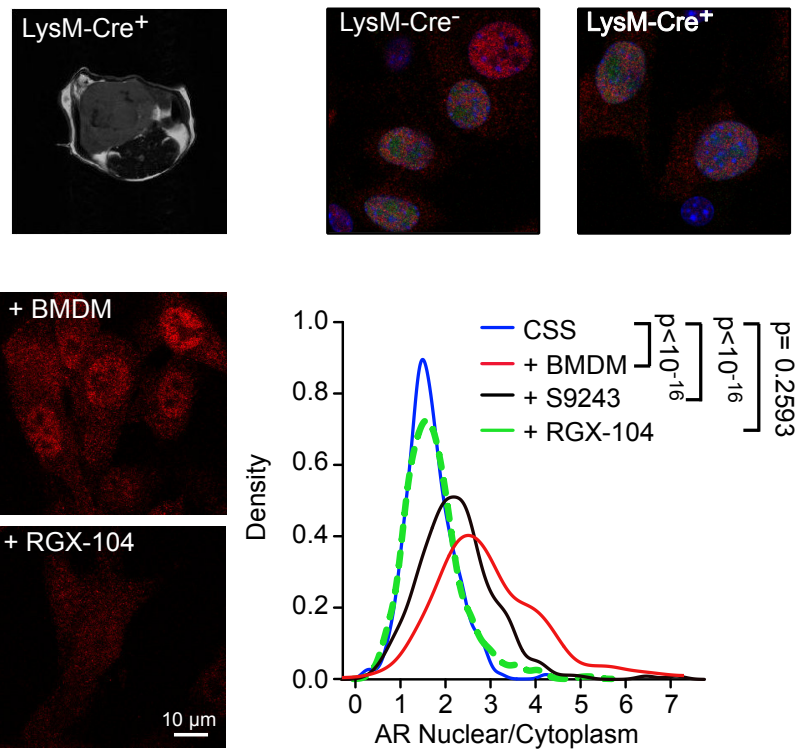

Figure 5 
bioRxiv preprint doi: https://doi.org/10.1101/2021.03.24.436480; this version posted March 24, 2021. The copyright holder for this preprint (which was not certified by peer review) is the author/funder. All rights reserved. No reuse allowed without permission.

A
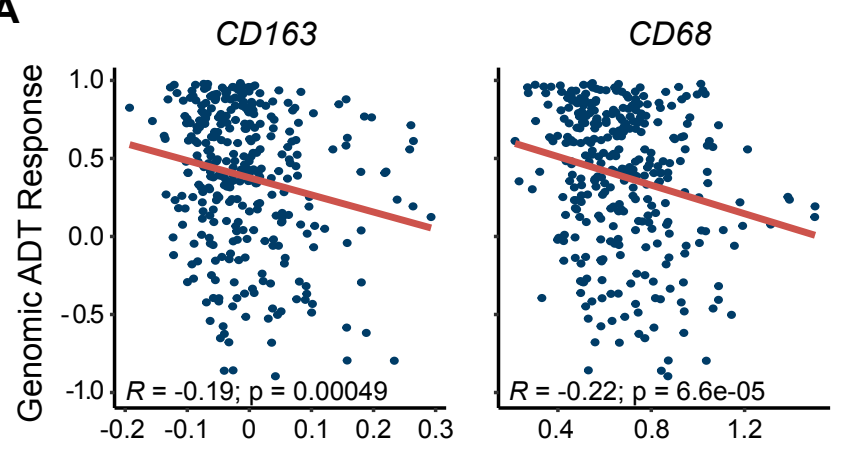

CSF1R

MRC1
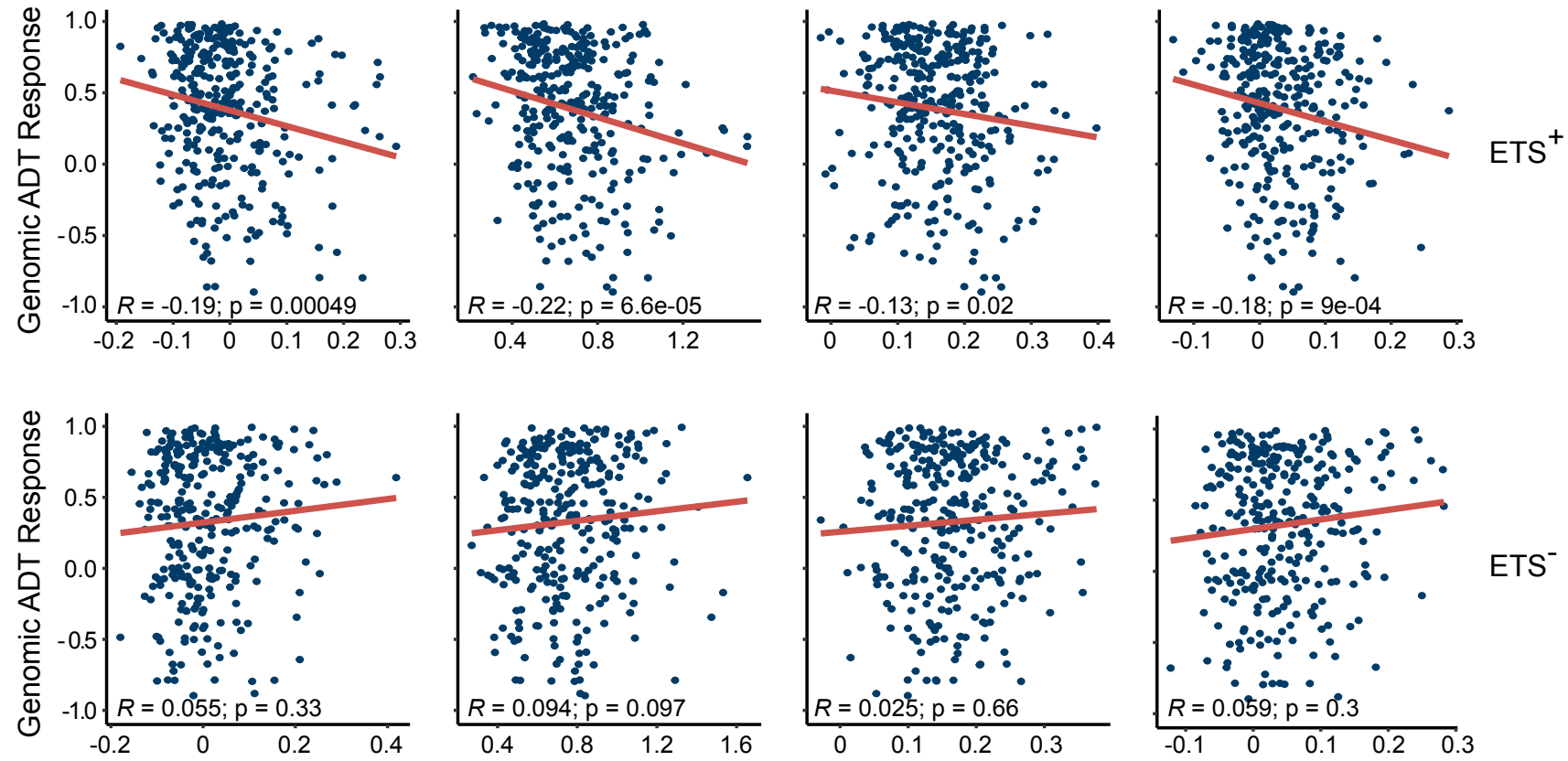

B

C

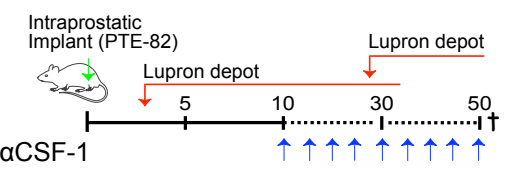

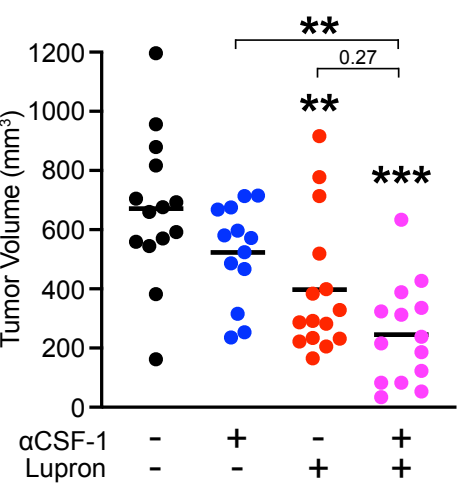

D

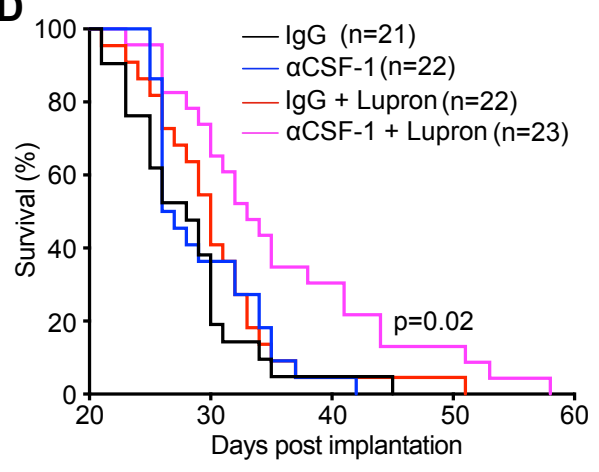

